\title{
Microscopic properties of xenon plasmas for density and temperature regimes of laboratory astrophysics experiments on radiative shocks
}

\author{
R. Rodríguez, ${ }^{1,2,{ }^{*}}$ G. Espinosa, ${ }^{1}$ J. M. Gil, ${ }^{1,2}$ C. Stehlé, ${ }^{3}$ F. Suzuki-Vidal, ${ }^{4}$ J. G. Rubiano, ${ }^{1,2}$ P. Martel,,${ }^{1,2}$ and E. Mínguez ${ }^{2}$ \\ ${ }^{1}$ Departamento de Física, Universidad de Las Palmas de Gran Canaria, 35003 Las Palmas de Gran Canaria, Las Palmas, Spain \\ ${ }^{2}$ Instituto de Fusión Nuclear, Universidad Politécnica de Madrid, 28040 Madrid, Spain \\ ${ }^{3}$ LERMA, Observatoire de Paris, UPMC, CNRSS, Meudon, 75014 Paris, France \\ ${ }^{4}$ The Blackett Laboratory, Imperial College, London SW7 2AZ, United Kingdom
}

(Received 17 March 2015; published 28 May 2015)

\begin{abstract}
This work is divided into two parts. In the first one, a study of radiative properties (such as monochromatic and the Rosseland and Planck mean opacities, monochromatic emissivities, and radiative power loss) and of the average ionization and charge state distribution of xenon plasmas in a range of plasma conditions of interest in laboratory astrophysics and extreme ultraviolet lithography is performed. We have made a particular emphasis in the analysis of the validity of the assumption of local thermodynamic equilibrium and the influence of the atomic description in the calculation of the radiative properties. Using the results obtained in this study, in the second part of the work we have analyzed a radiative shock that propagated in xenon generated in an experiment carried out at the Prague Asterix Laser System. In particular, we have addressed the effect of plasma self-absorption in the radiative precursor, the influence of the radiation emitted from the shocked shell and the plasma self-emission in the radiative precursor, the cooling time in the cooling layer, and the possibility of thermal instabilities in the postshock region.
\end{abstract}

DOI: 10.1103/PhysRevE.91.053106

PACS number(s): 52.25.Dg, 52.25.Os, 52.20.-j

\section{INTRODUCTION}

High-energy-density (HED) laboratory plasma astrophysics is a research field whose popularity has grown considerably over the past three decades. It deals with the experimental modeling of the astrophysical processes, involving studies of microphysics and large-scale flow phenomena [1]. Two developments in the field have contributed to the successful design of HED laboratory astrophysical experiments. First, it has been proved that the hydrodynamics can be scaled correctly between laboratory and astrophysical scenarios [2-7]. Second, the emergence of HED facilities, basically, power lasers and fast magnetic pinch machines ( $Z$ pinches) that allow matter to be placed in extreme states of temperature, density, and velocity, has enabled important research [4]. The laboratory experiments permit us to explain and predict what occurs in astrophysical phenomena and have the advantages of being repeatable and enabling control over the initial conditions. Moreover, they also provide data for verification and validation of several aspects of numerical codes such as atomic physics, hydrodynamics, equations of state, and radiative transfer. HED laboratory astrophysics includes, for example, experiments on radiative effects in shock waves, blast waves launched in atomic clusters media to emulate the ones observed in supernova remnants, and the formation of jets associated to newly forming stars. An extensive revision of these and other experiments can be found in Ref. [4].

One of the most interesting astrophysical phenomena is the shock waves which are ubiquitous throughout the universe and play a crucial role in the transport of energy into the interstellar medium [8]. When the radiation transport is important to the total energy budget, shock waves can be radiatively driven. Then the radiative energy flux and/or pressure plays an essential role

*Email address: rafael.rodriguezperez@ulpgc.es in the dynamics of a radiative shock [9]. At high shock velocity, the shocked medium is heated and ionized, emitting radiation, which produces radiative cooling. The radiation emitted heats and ionizes the unshocked medium ahead of the front shock, leading to the creation of a radiative precursor [10]. Therefore, the structure of a radiative shock includes a radiative precursor, a density jump, a cooling layer, and a final state [9]. Radiative shocks are observed around astronomical objects in a wide variety of forms, e.g., accretion shock, supernovae in their radiative cooling stage, bow shocks of stellar jet in galactic medium, and collision of interstellar clouds.

In laboratory astrophysics experiments, radiative shocks have been generated by driving a solid density plastic or beryllium piston into a xenon gas cell [4,11-18] by using a $\mathrm{kJ}$ laser to irradiate a pin or foil within a moderate- to high- $Z$ background gas $[19,20]$ or by depositing directly the energy of the laser into gas formed by atomic clusters [8,21-24]. For a given shock velocity and a given initial gas pressure, materials with high atomic numbers suit the achievement of the radiative regime, and for this reason, xenon is commonly used as the medium in which the radiative shock propagates. The electron temperatures and matter densities of many of these experiments are between 1 and $50 \mathrm{eV}$ and $10^{-3}$ and $10^{-1} \mathrm{~g} \mathrm{~cm}^{-3}$, respectively (free electron densities between $10^{18}$ and $10^{23} \mathrm{~cm}^{-3}$ ). For that range of plasma conditions the ion charge states that we have to consider are between $\mathrm{Xe}^{0+}$ and $\mathrm{Xe}^{+15}$. A multiply charged xenon structure is challenging since the ions contain $4 d$ and/or $5 p$ open shells, which introduces high complexity. In the works related to the radiative shock experiments cited previously, although radiative-hydrodynamics simulations are performed, there are no analyses of the influence, for instance, of the atomic or the population kinetic models used in the determination of the radiative properties. In Busquet et al. [25] a theoretical study of monochromatic and Rosseland mean opacities in the range of temperatures $5-50 \mathrm{eV}$ is made but only at one value of density 
of matter $\left(1 \mathrm{~g} \mathrm{~cm}^{-3}\right)$. In Rodriguez et al. [26] the authors made a study of the analysis of thermodynamic regimes of xenon plasmas as well as of the average ionization, charge-state distributions, and radiative power losses but for the range of matter densities of $10^{-5}-10^{-3} \mathrm{~g} \mathrm{~cm}^{-3}$. On the other hand, xenon is a popular material that can be used for extreme ultraviolet (EUV) sources at wavelengths near $13.5 \mathrm{~nm}$ for its use in EUV lithography, which is a promising technology in the microelectronics industry [27]. The plasma conditions in this case are in the range described above for the radiative shocks, and the most relevant charge stages of xenon are from $\mathrm{Xe}^{8+}$ to $\mathrm{Xe}^{13+}$. Due to the interest on the EUV lithography there are several theoretical and experimental works [28-32] that analyze the atomic description for that range of xenon ions but they are mainly focused on the calculation of the plasma emission in the range of wavelengths $10-20 \mathrm{~nm}$.

Therefore, as far as we know, there is no exhaustive study of microscopic properties of xenon plasmas in the range of conditions of interest of many of laboratory astrophysics experiments on radiative shocks and also of the influence of several issues on their calculations, such as the population kinetic model, atomic description, plasma self-absorption, or external radiation fields. These plasma properties (such as, for example, the average ionization, charge state distribution, opacities, and emissivities) are key ingredients in radiative-hydrodynamic simulations or to interpret experimental spectra. These facts have motivated this work. Accordingly, this work is organized as follows. In Sec. II we present the theoretical model used to calculate the microscopic properties of xenon plasmas. Furthermore, in order to show the accuracy of our model, comparisons with calculations performed with other codes for some properties of xenon plasmas are made. Section III is devoted to the analysis of the influence of the nonlocal thermodynamic equilibrium (NLTE) effects on the simulation of plasma-level populations and radiative properties. This analysis is made under the detailed configuration accounting (DCA) approach, which is sufficient for this purpose. In the following section, the influence of the atomic description, DCA, or detailed level accounting (DLA) approach and configuration mixing, in the calculation of the plasma radiative properties, is addressed. In Sec. V, for a particular astrophysical laboratory experiment on radiative shocks [33], we make a study of the effect of plasma self-absorption and external radiation field in the calculation of the microscopic properties of the plasma in the radiative precursor. Furthermore, the determination of the ranges of plasma conditions in the cooling region in which thermal instabilities could occur is also addressed. Finally, in Sec. VI the conclusions are presented.

\section{MODEL DESCRIPTION}

The calculation of plasma microscopic properties such as the average ionization or the opacity requires atomic data, such as energy levels and oscillator strengths, and atomic-level populations. In the following the models employed in this work to calculate them are described.

\section{A. Atomic structure}

The calculation of the atomic structure and photoionization cross sections have been carried out using the FAC code [34]. In this code a fully relativistic approach based on the Dirac equation is used. Thus, in the calculation of the atomic structure, the atomic levels of an atomic ion are obtained by diagonalizing the relativistic Hamiltonian. The photoionization cross section is calculated using a relativistic distorted-wave approximation. FAC is able to work either under the DCA or DLA approaches. For the former, the transition energies include the unresolved transition array (UTA) [35] shifts and a correction to the oscillator strengths due to the configuration interaction (CI) within the same nonrelativistic configurations. In the DLA approach, FAC can include different levels of CI.

In this work the study has been made mainly in the DCA approach although there is a section devoted to make a qualitative analysis of the influence of the atomic description (i.e., DCA against DLA and CI effects) in the calculation of the radiative properties in the plasma conditions of interest in this work. For that range of plasma conditions the ions of xenon found are between $\mathrm{Xe}^{0+}$ and $\mathrm{Xe}^{13+}$, as we will show in a further section. So we take $\mathrm{Xe}^{3+}$ and $\mathrm{Xe}^{10+}$ as examples to illustrate the atomic configurations included in this work in the DCA approach. For the former ion, the ground configuration is $[\mathrm{Kr}] 4 d^{10} 5 s^{2} 5 p^{3}$ and the excited configurations considered were the following: $5(s, p)^{4} 5 l$ (with $l=d, f, g$ ), where $(s, p)^{4}$ denotes all the configurations that arise from all the possible distributions of the four electrons in subshells $5 s$ and $5 p$, $5(s, p)^{3} 5 d^{2}, 5(s, p)^{4} 4 f^{1}, 5(s, p)^{4} n^{\prime} l^{\prime}$ (with $n^{\prime}=6-10$ and $\left.l^{\prime}=s-f\right), 5(s, p)^{3} 5 d^{1} 4 f^{1}, 5(s, p)^{3} 5(f, g)^{1}, 5(s, p)^{3} 5 d^{1} n^{\prime} l^{\prime}$, and $4 d^{9} 5(s, p)^{4}$. The ground configuration of $\mathrm{Xe}^{10+}$ is $[\mathrm{Kr}] 4 d^{8}$, and the excited configurations included were $4(s, p)^{7} 4 d^{9}, \quad 4(s, p)^{6} 4 d^{10}, 4(s, p)^{7} 4 d^{8} 4 f^{1}, \quad 4(s, p)^{6} 4 d^{9} 4 f^{1}$, $4(s, p)^{5} 4 d^{10} 4 f^{1}, \quad 4(s, p)^{8} 4 d^{6} 4 f^{2}, \quad 4(s, p)^{7} 4 d^{7} 4 f^{2}$, $4(s, p)^{6} 4 d^{8} 4 f^{2}, \quad 4(s, p)^{5} 4 d^{9} 4 f^{2}, \quad 4(s, p)^{4} 4 d^{10} 4 f^{2}, \quad 4 d^{7} n l$, $4(s, p)^{7} 4 d^{8} n l, 4 d^{6} 4 f^{1} n l$ (with $n=5-10$ and $l=s-f$ ), and $4 d^{6} n^{\prime} l^{\prime} n^{\prime \prime} l^{\prime \prime}$ (with $n^{\prime}, n^{\prime \prime}=5,6$ and $l^{\prime}, l^{\prime \prime}=s-f$ ).

\section{B. Calculation of plasma atomic-level populations}

At high densities, when the plasma approaches the local thermodynamic equilibrium (LTE), the population of different ionization stages, $N_{\varsigma}$, can be obtained by solving the Saha equation,

$$
\frac{N_{\zeta+1} n_{e}}{N_{\zeta}}=\frac{Z_{e} Z_{\zeta+1}}{Z_{\zeta}} e^{-\left(I_{\zeta}-\Delta I_{\zeta}\right) / k T_{e}},
$$

where $n_{e}$ is the free electrons density, $Z_{e}$ and $Z_{\zeta}$ are the partition functions of free electrons and ion $\zeta$, respectively, $I_{\zeta}$ is the ionization potential of the ionization stage $\zeta$, and $\Delta I_{\zeta}$ is the depression of the ionization potential (continuum lowering, CL) due to the plasma environment. In this work we apply the formulation developed by Stewart and Pyatt [36]. The application of the CL can restrict the number of bound states available. The Boltzmann distribution function is used to calculate the population of each atomic level belonging to the ionization stage $\zeta$.

For arbitrary densities the atomic-level populations are determined from the solution of a system of collisionalradiative (CR) rate equations. This set of kinetic rates equations 
is given by

$$
\frac{d N_{\zeta i}(\mathbf{r}, t)}{d t}=\sum_{\zeta^{\prime} j} N_{\zeta^{\prime} j}(\mathbf{r}, t) R_{\zeta^{\prime} j \rightarrow \zeta i}^{+}-\sum_{\zeta^{\prime} j} N_{\zeta i}(\mathbf{r}, t) R_{\zeta i \rightarrow \zeta^{\prime} j}^{-},
$$

where $N_{\zeta i}$ is the population density of the atomic configuration or level (depending on the atomic approach) $i$ of the ion with charge state $\zeta$. The terms $R_{\zeta^{\prime} j \rightarrow \zeta i}^{+}$and $R_{\zeta^{\prime} j \rightarrow \zeta i}^{-}$take into account all the atomic processes, both collisional and radiative, which contribute to populate and depopulate the state $\zeta i$, respectively. Two complementary equations which have to be satisfied together with Eq. (2) are, first, the requirement that the sum of all the partial densities equals the total ion density, $n_{\text {ion }}$,

$$
\sum_{\zeta=0}^{Z} \sum_{i=0}^{M_{\zeta}-1} N_{\zeta i}=n_{\mathrm{ion}}
$$

and, second, the charge neutrality condition in the plasma,

$$
\sum_{\zeta=0}^{Z} \sum_{i=0}^{M_{\zeta}-1} \zeta N_{\zeta i}=n_{e}
$$

where $M_{\zeta}$ is the total number of levels for the charge state $\zeta$. The plasma average ionization is defined as

$$
\bar{Z}=\frac{\sum_{\zeta=0}^{Z} \zeta N_{\zeta}}{\sum_{\zeta=0}^{Z} N_{\zeta}}=\frac{n_{e}}{n_{\text {ion }}},
$$

and the plasma charge state distribution (CSD) is defined as the set of the population densities, $\left(N_{\zeta}\right)$, of the ions present in the plasma for a given condition of density and temperature and which is obtained from the resolution of either the rate or Saha equations.

The set of rate equations given by Eq. (2) are coupled to the radiative transfer equation

$$
\begin{aligned}
\frac{1}{c} & \frac{\partial I(\mathbf{r}, t, v, \mathbf{e})}{\partial t}+\mathbf{e} \cdot \nabla I(\mathbf{r}, t, v, \mathbf{e}) \\
& =-\kappa(\mathbf{r}, t, v) I(\mathbf{r}, t, v, \mathbf{e})+j(\mathbf{r}, t, v),
\end{aligned}
$$

where $I$ is the specific intensity, $v$ the photon frequency, and $\mathbf{e}$ a unitary vector in the direction of the radiation propagation. The emissivity and the absorption coefficients $[j(\mathbf{r}, t, v)$ and $\kappa(\mathbf{r}, t, v)$, respectively] couple the radiative equation with the rate equations.

The CR (for steady-state situations, CRSS) as well as the Saha-Boltzmann (SB) equations, used in this work are implemented in ABAKO code [37]. The atomic processes included in the CRSS are collisional ionization [38] and three-body recombination, spontaneous decay [34], collisional excitation [39] and deexcitation, radiative recombination [40], autoionization, and electron capture (obtained from the collisional excitation cross section using a known approximation [41]). We have added between brackets the references from which their approximated analytical rates coefficients have been acquired. The rates of the inverse processes are obtained through the detailed balance principle. In order to take into account the effect of external radiation fields in the calculation of the atomic-level populations, the radiative-driven processes photoexcitation, photodeexcitation, and photo-ionization are considered in the $\mathrm{CR}$ model. For the latter the Kramers photoionization cross section [40] was used. The rates of these processes are obtained assuming that the intensity of the radiation field can be modeled with a diluted Planck function. The same formalism of CL as for SB equations is included in the $\mathrm{CR}$ model. Because of the inclusion of the CL, both the rate and SB equations must be solved iteratively, since the former depends on the average ionization. In ABAKO it is assumed that the system has had enough time to thermalize and, therefore, both the electrons and ions have a Maxwell-Boltzmann type energy distribution. Furthermore, in ABAKO it is also assumed that electron and ion temperatures are equal.

Finally, plasma self-absorption (i.e., opacity effects) can be also included in the CR model of ABAKO. This effect is modeled in an approximate way using the escape factor formalism for the bound-bound opacity. To compute the escape factors for the three basic geometries (planar, cylindrical, and spherical), the technique described in Ref. [42] was adopted, assuming a uniform distribution for emitting atoms and isotropic emission. This formalism avoids the need to perform a simultaneous calculation of radiative transport and atomic physics. Due to this fact and as the intensity of the external radiation field for the radiative-driven processes is modeled by a Planck function, in ABAKO the radiative transfer and rate equations are uncoupled.

\section{Calculation of the plasma radiative properties}

Plasma radiative properties are calculated using the RAPCAL code $[43,44]$. The monochromatic emissivity and absorption are denoted in this work as $j(v)$ and $\kappa(v)$, respectively (where we have omitted the dependence on the position and time to simplify the notation) and both of them include the boundbound, bound-free, and free-free contributions

$$
\begin{aligned}
& j(v)=j_{\mathrm{bb}}(v)+j_{\mathrm{bf}}(v)+j_{\mathrm{ff}}(v), \\
& \kappa(v)=\kappa_{\mathrm{bb}}(v)+\kappa_{\mathrm{bf}}(v)+\kappa_{\mathrm{ff}}(v),
\end{aligned}
$$

where $v$ is the photon frequency. The bound-bound contribution to the emissivity is given by

$$
j_{\mathrm{bb}}(v)=\sum_{\zeta} \sum_{i, j} j_{\zeta j \rightarrow \zeta i}(v),
$$

with

$$
j_{\zeta j \rightarrow \zeta i}(v)=\frac{h v}{4 \pi} N_{\zeta j} A_{\zeta j \rightarrow \zeta i} \phi_{i j}(v),
$$

where $A_{\zeta j \rightarrow \zeta i}$ is the Einstein coefficient for spontaneous deexcitation between the bound states $j, i$ of the ion $\zeta$ and $h$ is the Planck's constant. The radiative transitions rates in FAC are calculated in the single-multipole approximation, and in this work they were obtained by use of the electric dipole approach. The bound-bound contribution to the absorption is given by

$$
\kappa_{\mathrm{bb}}(v)=\sum_{\zeta} \sum_{i, j} \kappa_{\zeta i \rightarrow \zeta j}(v)
$$

with

$$
\kappa_{\zeta i \rightarrow \zeta j}(v)=\frac{h v}{4 \pi} N_{\zeta i} \frac{g_{\zeta j}}{g_{\zeta i}} \frac{c^{2}}{2 h v_{i j}^{3}} A_{\zeta j \rightarrow \zeta i} \phi_{i j}(\nu)\left(1-\frac{g_{\zeta i}}{g_{\zeta j}} \frac{N_{\zeta j}}{N_{\zeta i}}\right),
$$


where $c$ is the speed of light and $g_{\zeta i}$ and $g_{\zeta j}$ are the statistical weights of the $i$ and $j$ levels. In the previous equations, $\phi_{i j}(v)$ represents the line profile both for line emission and absorption since in this work complete redistribution hypothesis is assumed [45]. In the evaluation of the line profile, natural, Doppler, and electron-impact [46] broadenings were included as was the UTA width in the DCA approach. The line-shape function is applied with the Voigt profile that incorporates all these broadenings.

The bound-free contribution to the emissivity is determined by means of

$$
j_{\mathrm{bf}}(v)=\sum_{\zeta, i} \sum_{\zeta, j} j_{\zeta+1 j \rightarrow \zeta i}(v)
$$

with

$$
\begin{aligned}
j_{\zeta+1 j \rightarrow \zeta i}(\nu)= & \frac{h}{2 \pi c^{2}}\left(\frac{1}{2 m_{e}}\right)^{3 / 2} N_{\zeta+1 j} n_{e} f(\varepsilon) \frac{g_{\zeta i}}{g_{\zeta+1 j}} \\
& \times \frac{(h v)^{3}}{\varepsilon^{1 / 2}} \sigma_{\zeta i \rightarrow \zeta+1 j}^{\text {pho }}(\nu),
\end{aligned}
$$

with $\varepsilon$ the energy of the free electron and $m_{e}$ the electron mass. As noted above, in ABAKO a Maxwell-Boltzmann distribution, $f(\varepsilon)$, at temperature $T_{e}$ for the free electrons is assumed. Moreover, the photoionization cross section, $\sigma_{\zeta i \rightarrow \zeta+1 j}^{\text {pho }}(v)$, has been calculated using the FAC code in the relativistic distorted-wave approach. For the bound-free spectra the DCA approximation for the atomic description has been used as well. The bound-free contribution to the absorption is given by

$$
\kappa_{\mathrm{bf}}(v)=\sum_{\zeta, i} \sum_{\zeta, j} \kappa_{\zeta i \rightarrow \zeta+1 j}(v)
$$

with

$$
\kappa_{\zeta i \rightarrow \zeta+1 j}(\nu)=N_{\zeta i} \sigma_{\zeta i \rightarrow \zeta+1 j}^{\mathrm{pho}}(\nu)\left[1-\frac{N_{\zeta+1 j} n_{e} f(\varepsilon) g_{\zeta i}}{N_{\zeta i} g_{\zeta+1 j} g(\varepsilon)}\right],
$$

where $g(\varepsilon)$ is the density of states with energy $\varepsilon$ which, assuming an ideal gas of free electrons, is given by

$$
g(\varepsilon)=4 \pi\left(\frac{2 m_{e}}{h^{2}}\right)^{3 / 2} \varepsilon^{1 / 2} .
$$

For the free-free contributions to the emissivity and the absorption the Kramers semi-classical expression for the inverse bremsstrahlung cross section has been used [47]

$$
\sigma_{\zeta}^{i b r}(v)=\frac{16 \pi^{2} e^{2} h^{2} \alpha}{3 \sqrt{3}\left(2 \pi m_{e}\right)^{3 / 2}} \frac{\zeta^{2} n_{e}}{T_{e}^{1 / 2}(h v)^{3}} .
$$

Assuming a Maxwell-Boltzmann distribution for the free electrons, we obtain

$$
\begin{array}{r}
j_{\mathrm{ff}}(v)=\frac{32 \pi^{2} e^{4} a_{0}^{2} \alpha^{3}}{\sqrt{3}\left(2 \pi m_{e}\right)^{3 / 2} h}\left(\frac{m_{e}}{2 \pi T_{e}}\right)^{1 / 2} \overline{Z^{2}} n_{\mathrm{ion}} n_{e} e^{-h v / T_{e}}, \\
\kappa_{\mathrm{ff}}(v)=\frac{16 \pi^{2} e^{2} h^{2} \alpha}{3 \sqrt{3}\left(2 \pi m_{e}\right)^{3 / 2}} \frac{\overline{Z^{2}} n_{\mathrm{ion}} n_{e}}{T_{e}^{1 / 2}(h v)^{3}}\left(1-e^{-h v / T_{e}}\right) .
\end{array}
$$

In order to determine the opacity, $k(v)$, the absorption due to the scattering of photons is also taken into account. In RAPCAL this one is approximated using the Thomson scattering cross section [48],

$$
\kappa_{\text {scatt }}=n_{e} \sigma^{\text {Thom }},
$$

with $\sigma^{\text {Thom }}=6.65 \times 10^{-25} \mathrm{~cm}^{2}$.

Finally, the opacity is given by

$$
k(v)=\frac{1}{\rho}\left[\kappa(v)+\kappa_{\text {scatt }}\right],
$$

with $\rho$ the density of matter. The source function is then obtained as

$$
S(v)=\frac{j(v)}{\rho k(v)} .
$$

As noted above, RAPCAL also provides the Planck, $k_{P}$, and Rosseland, $k_{R}$, mean opacities, which are given by [49]

$$
\begin{gathered}
k_{P}=\int_{0}^{\infty} d \nu \tilde{B}(\nu, T)\left[k(\nu)-\kappa_{\text {scatt }} / \rho\right], \\
\frac{1}{k_{R}}=\int_{0}^{\infty} d \nu \frac{\partial \tilde{B}(\nu, T)}{\partial T} \frac{1}{k(\nu)},
\end{gathered}
$$

where $\tilde{B}(\nu, T)$ is the normalized Planckian function,

$$
\tilde{B}(\nu, T)=\frac{15}{\pi^{4} T} \frac{u^{3}}{e^{u}-1}, \quad u=\frac{h v}{T} .
$$

The radiative power loss (RPL) is evaluated as follows [50]. For the bound-bound contribution,

$$
P_{\mathrm{bb}}=\sum_{\zeta} \sum_{i j} h v_{i j} A_{i j} N_{\zeta j} .
$$

The bound-free contribution is given by

$$
\begin{aligned}
P_{\mathrm{bf}}= & 4 \pi \sum_{\zeta} \sum_{i j} N_{\zeta i}\left(\frac{N_{\zeta+1 j} N_{\zeta}}{N_{\zeta i} N_{\zeta+1}}\right)^{\mathrm{LTE}} \int_{\nu_{0}}^{\infty} \sigma_{\zeta i \rightarrow \zeta+1 j}^{\mathrm{pho}}(v) \\
& \times\left(\frac{2 h \nu^{3}}{c^{2}}\right) e^{-h v / T_{e}} d \nu,
\end{aligned}
$$

where $v_{0}$ is the threshold energy of the $\zeta i \rightarrow \zeta+1 j$ transition and the LTE population ratio is obtained from the Saha equation. The contribution from the free-free transitions is given for a pure Coulomb field (in $\mathrm{eV} \mathrm{s}^{-1} \mathrm{~cm}^{-3}$ ) as follows [51]:

$$
P_{\mathrm{ff}}=9.55 \times 10^{-14} n_{e} T_{e}^{1 / 2} \sum_{\zeta} Z_{\zeta}^{2} N_{\zeta},
$$

where the gaunt factor has been assumed equal to unity. The total RPL is then obtained as the sum of the three contributions.

\section{Comparisons with other models}

The ABAKO and RAPCAL codes have been successfully tested with experimental results and numerical simulations for plasmas of both low- and high- $Z$ elements either under LTE or NLTE conditions in optically thin and thick (homogeneous and nonhomogeneous) situations [37,43,44,52]. Here we present comparisons of some plasma properties for xenon in density and temperature conditions of interest in this work in order to show the accuracy of our calculations in these conditions. In particular, the average ionization, CSD, and radiative power loss are compared. 
TABLE I. Comparison of the average ionization at several temperatures and at an electron density of $10^{18} \mathrm{~cm}^{-3}$, with calculations of codes from the fourth non-LTE code comparison workshop [53].

$T_{e}(\mathrm{eV})$ This work Code 1 Code 2 Code 3 Code 4 Code 5 Code 6

\begin{tabular}{rrrrrrrr}
\hline 10 & 6.86 & 6.71 & 7.36 & 6.96 & 6.89 & 6.85 & 6.76 \\
20 & 8.60 & 9.12 & 8.53 & 8.13 & 8.80 & 8.92 & 7.82 \\
50 & 11.47 & 11.92 & 13.58 & 10.70 & 11.37 & 12.60 & 12.26 \\
\hline \hline
\end{tabular}

Table I shows a comparison of the average ionization for a plasma of xenon at three temperatures $(10,20,50 \mathrm{eV})$ and an electron density of $10^{18} \mathrm{~cm}^{-3}$ with calculations performed by NLTE kinetic codes from the fourth non-LTE code comparison workshop [53]. From the table we observe that the agreement among the codes is better for the first temperatures and this worsens for the largest temperature. This fact could be due to the fact that at $50 \mathrm{eV}$ the average ionization is between 11 and 12 and therefore the $4 d$ shell is open, which leads to many more possible atomic levels than for the other two lower temperatures, in which the shell is almost fully occupied. Since the atomic description and the atomic configurations included in each code differ, it is expected that the discrepancies are greater at this temperature. In any case, we can observe that our simulation agrees with most of the codes for the two lowest temperatures. This result can also be observed in Fig. 1, where we have represented the comparison of the CSDs among the codes for these two temperatures. At $50 \mathrm{eV}$, where the differences among the codes are greater, our simulation provides an average ionization which is in the interval 11-12 as well as the calculations of most of the codes.

In Table II we present a comparison of the average ionization and CSD for two plasma conditions of an experiment of a laser-produced xenon plasma related to its application for EUV lithography [28]. In this case, the results reported by the authors were obtained using the SCO code [54] based on the superconfiguration (SC) approach $[55,56]$ assuming LTE. We detect a good agreement in the average ionization and also in the CSD at $26 \mathrm{eV}$. At $30 \mathrm{eV}$ there are some differences in the fractional abundance mainly in the three most charged ions. In both simulations the set of configurations and the atomic description used (DCA or SC approaches) differ and these facts can introduce some discrepancies in the results. However, there is an agreement in both calculations in the three most abundant ions, the ions with fractional abundances greater than $1 \%$, and the behavior of the CSD.

In Table III we present a comparison of the average ionization and CSD with a theoretical analysis carried out by Sasaki et al. [31] using the HULLAC code [57] to calculate the atomic structure, combining DCA and fine structure approaches, and assuming LTE to calculate the ion populations. Our calculation provides a quite similar average ionization. With respect to the $\mathrm{CSD}$, as was already the case for the previous comparison, the differences between the atomic models and the configurations considered are responsible for the discrepancies. Even so, there are agreements in the behavior of the CSD and the ions with fractional abundance greater than the $1 \%$ and also in the fractional abundance of the most abundant ion.

Finally, we have compared the radiative energy loss coefficients [58], i.e., power radiated per ion and per electron,
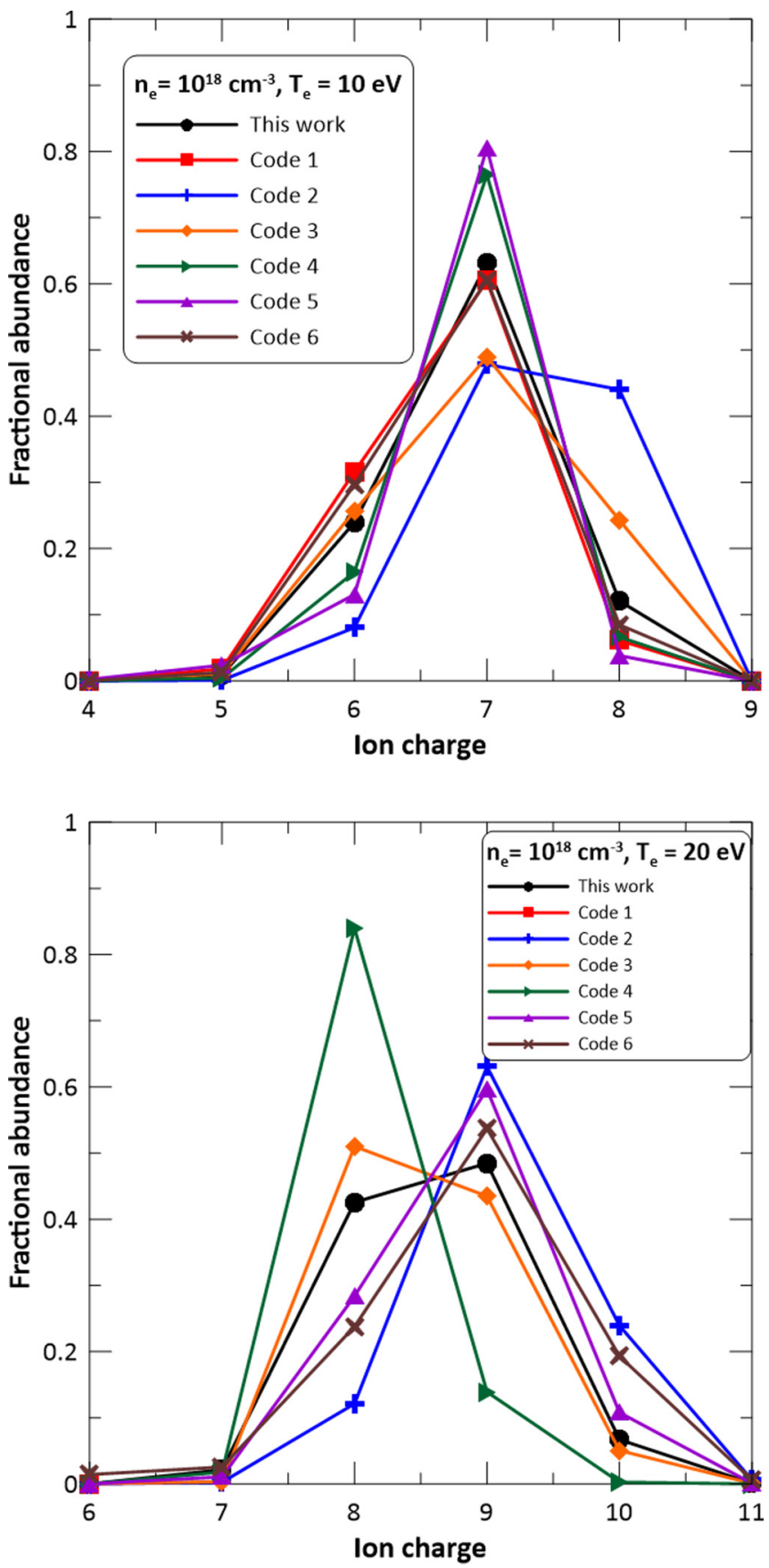

FIG. 1. (Color online) Comparison of the charge state distributions at two temperatures with calculations of codes from the fourth non-LTE code comparison workshop [53].

with experimental values obtained when xenon gas was introduced at small impurity concentration to high-density hydrogen plasma produced in a gas-liner pinch [59]. The plasma conditions in this case are in the range of interest in this work. The estimated accuracy of the experimental energy-loss coefficients was 2. The comparison is shown in Table IV. From the table we observe that, except for the second and third cases, our theoretical calculations are of the same order of magnitude of the experimental calculations, even in the DCA approach that we are using in this comparison. 
TABLE II. Comparison of the average ionization and charge state distributions (in percentages) with calculations of Gilleron et al. [28] for a density of matter of $10^{-2} \mathrm{~g} \mathrm{~cm}^{-3}$.

\begin{tabular}{lcclcc}
\hline \hline & \multicolumn{2}{c}{$T_{e}=26 \mathrm{eV}$} & & \multicolumn{2}{c}{$T_{e}=30 \mathrm{eV}$} \\
\cline { 2 - 3 } \cline { 5 - 6 } & This work & Gilleron et al. & & This work & Gilleron et al. \\
\hline $\bar{Z}$ & 9.76 & 9.68 & & 10.55 & 10.62 \\
$\mathrm{Xe}^{8+}$ & 7.6 & 6.5 & & 1.4 & 2.0 \\
$\mathrm{Xe}^{9+}$ & 31.3 & 32.2 & & 12.4 & 11.4 \\
$\mathrm{Xe}^{10+}$ & 38.8 & 39.3 & & 30.4 & 29.7 \\
$\mathrm{Xe}^{11+}$ & 19.9 & 19.1 & & 42.1 & 35.7 \\
$\mathrm{Xe}^{12+}$ & 2.0 & 2.4 & & 12.5 & 17.7 \\
$\mathrm{Xe}^{13+}$ & - & - & & 1.1 & 3.2 \\
\hline \hline
\end{tabular}

RPL, and then the radiative energy loss coefficient, is more sensitive to the atomic-level populations in the plasma than the average ionization and the CSD. Then it seems that the atomic description selected for the population kinetics as well as the atomic configurations included will strongly affect the RPL.

Therefore, from the whole set of comparisons presented, both in LTE and NLTE, for xenon plasmas in the range of plasma conditions of interest of this work, we can conclude that our atomic and population kinetic models are accurate enough for the qualitative analysis of different effects in the calculation of the xenon plasma microscopic properties that we present in this work.

\section{NLTE AND LTE ANALYSIS}

In a previous paper [60] the thermodynamic regime of optically thin plasmas in the stationary situation was analyzed for a wide range of electron temperatures (1-1100 eV) and matter densities $\left(10^{-5}-10^{2} \mathrm{~g} \mathrm{~cm}^{-3}\right)$. However, in that work the influence of NLTE or LTE simulations in the calculation of plasma microscopic properties was not analyzed for the range of plasma conditions of interest in this work. In order to analyze the NLTE effects we will compare the results obtained for the different properties using the SB and the rate equations of the CRSS model implemented in ABAKO. We consider that for the purpose of this section, which is mainly to give indications about the relevance of the NLTE effects, the DCA approach for the atomic description is enough. We started

TABLE III. Comparison of the average ionization and chargestate distributions (in percentages) with calculations of Sasaki et al. [31] for an electron temperature of $25 \mathrm{eV}$ and electron density of $10^{21} \mathrm{~cm}^{-3}$.

\begin{tabular}{lcc}
\hline \hline & This work & Sasaki et al. \\
\hline $\bar{Z}$ & 8.59 & 8.70 \\
$\mathrm{Xe}^{6+}$ & 1.4 & 1.5 \\
$\mathrm{Xe}^{7+}$ & 6.1 & 7.2 \\
$\mathrm{Xe}^{8+}$ & 39.0 & 32.0 \\
$\mathrm{Xe}^{9+}$ & 40.0 & 41.0 \\
$\mathrm{Xe}^{10+}$ & 12.0 & 16.0 \\
$\mathrm{Xe}^{11+}$ & 1.5 & 1.9 \\
\hline \hline
\end{tabular}

TABLE IV. Comparison of the radiative-energy loss coefficients (in $\mathrm{Wm}^{-3}$ ) with experimental values reported by Baig and Kunze [59].

\begin{tabular}{lcccc}
\hline \hline$T_{e}(\mathrm{eV})$ & $n_{e}\left(\mathrm{~cm}^{-3}\right)$ & \% of Xe & This work & Baig and Kunze \\
\hline 12.5 & $1.8 \times 10^{18}$ & 0.06 & $0.80 \times 10^{-32}$ & $1.70 \times 10^{-32}$ \\
10.0 & $1.3 \times 10^{18}$ & 0.06 & $0.26 \times 10^{-32}$ & $1.40 \times 10^{-32}$ \\
7.5 & $0.8 \times 10^{18}$ & 0.06 & $0.31 \times 10^{-32}$ & $1.90 \times 10^{-32}$ \\
10.0 & $2.0 \times 10^{18}$ & 0.10 & $0.30 \times 10^{-32}$ & $0.80 \times 10^{-32}$ \\
7.5 & $1.1 \times 10^{18}$ & 0.10 & $0.20 \times 10^{-32}$ & $0.70 \times 10^{-32}$ \\
7.5 & $2.3 \times 10^{18}$ & 0.40 & $0.46 \times 10^{-32}$ & $0.60 \times 10^{-32}$ \\
\hline \hline
\end{tabular}

analyzing the average ionization, CSDs, and plasma-atomiclevel populations. Figure 2 displays a comparison between the average ionizations obtained with both models. In the figure, the comparison is shown for all the temperatures of the range and for three matter densities, the ones of the limits and the intermediate one. From the figure we detect that for the highest density considered $\left(10^{-1} \mathrm{~g} \mathrm{~cm}^{-3}\right)$ the agreement is excellent for the whole range of temperatures. We have obtained that this good agreement is also retained for the CSDs obtained using both models. For this density of matter, the ions present in the plasma in the range of temperatures $1-50 \mathrm{eV}$ are those from $\mathrm{Xe}^{0+}$ to $\mathrm{Xe}^{13+}$. For the intermediate-density case $\left(10^{-2} \mathrm{~g} \mathrm{~cm}^{-3}\right)$ we observe a noticeable agreement between the SB and CRSS results for temperatures lower than $40 \mathrm{eV}$, and even at larger temperatures, where some slight differences are detected, the relative differences are always lower than $5 \%$. At this density of matter the ions that contribute in the range of electron temperatures are those from $\mathrm{Xe}^{0+}$ to $\mathrm{Xe}^{15+}$. The diminution of the density of matter yields to a decrease in the plasma recombination and therefore the range of charge stages is larger at $10^{-2}$ than at $10^{-1} \mathrm{~g} \mathrm{~cm}^{-3}$. With respect to the CSDs provided by both models in this

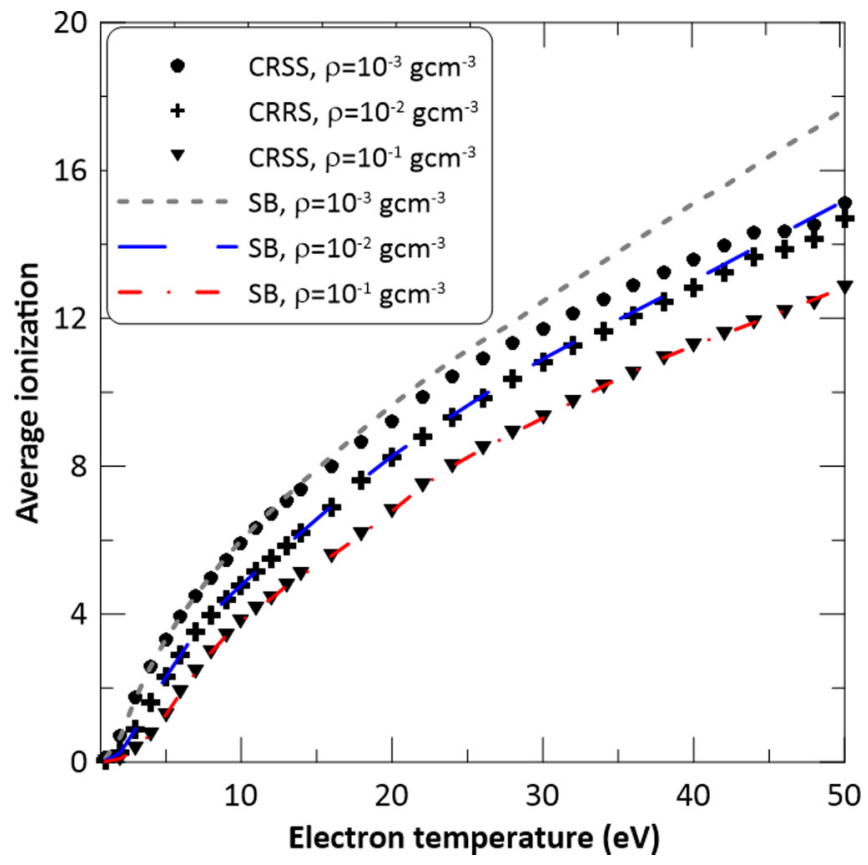

FIG. 2. (Color online) Comparison of the average ionizations obtained using SB and CRSS models. 
intermediate density we obtained that they are quite similar in the whole range of temperatures. Finally, at the lower density of matter of the range $\left(10^{-3} \mathrm{~g} \mathrm{~cm}^{-3}\right)$, we can detect slight differences for temperatures larger than $20 \mathrm{eV}$, although the relative differences are greater than $5 \%$ only for temperatures higher than $30 \mathrm{eV}$. For a fixed density of matter, as the temperature increases the departure of the plasma from LTE is larger. These differences in the average ionization will be more noticeable in the CSD since the latter is a less average quantity than the former. Figure 3 displays the comparison between the CSDs calculated using both population kinetic
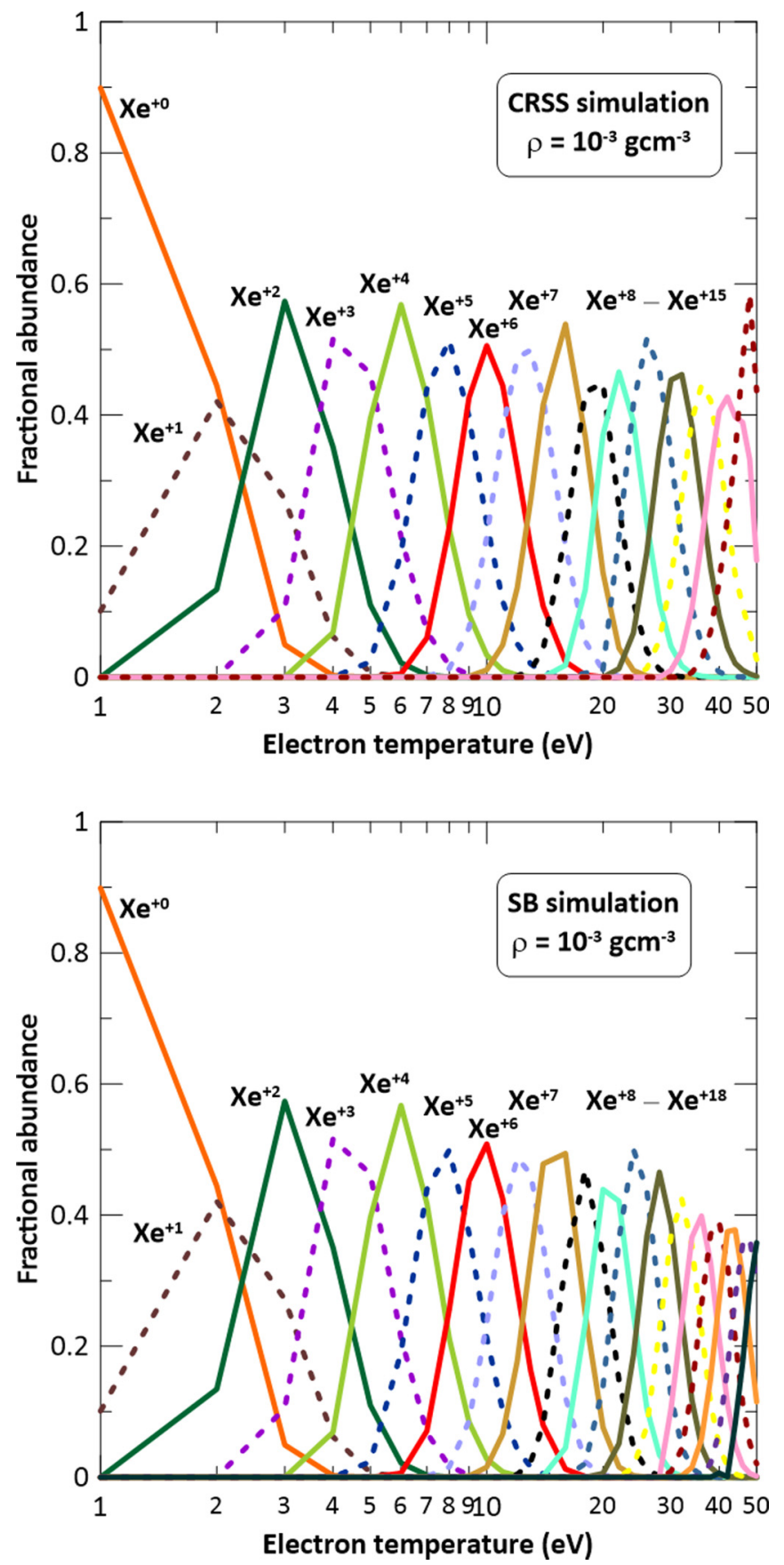

FIG. 3. (Color online) Comparison of the CSD using SB and CRSS models as a function of the electron temperature and for a density of matter of $10^{-3} \mathrm{~g} \mathrm{~cm}^{-3}$. models for this density of matter. The differences in the fractional abundances are beginning to emerge for $\mathrm{Xe}^{8+}$ ions at temperatures around $15 \mathrm{eV}$, although they are still small. However, for the average ionization, as noted above, the differences were almost undetectable until temperatures higher than $20 \mathrm{eV}$. The differences between the CSDs continue to grow with the temperature, and even for the highest temperatures $(35-50 \mathrm{eV})$ there are ion charge stages in the $\mathrm{SB}$ simulation $\left(\mathrm{Xe}^{16+}-\mathrm{Xe}^{18+}\right)$ that are not present in the CRSS simulation. This result is connected to the fact that, of the LTE, the SB equations overstimate the average ionization, as Fig. 2 shows. Therefore, LTE could be assumed for the calculation of the average ionization and CSDs for matter densities between $10^{-2}$ and $10^{-1} \mathrm{~g} \mathrm{~cm}^{-3}$, whereas for densities between $10^{-1}$ and $10^{-2} \mathrm{~g} \mathrm{~cm}^{-3}$ this approach is accurate enough for electron temperatures lower than $20 \mathrm{eV}$. For higher temperatures, a NLTE simulation might be necessary.

Plasma radiative properties are more sensitive to the population kinetic model used than the previous properties, since the former directly depends on the plasma-level populations, whereas average ionization and CSD are averaged. Therefore, the analysis of the population kinetic model on the level populations will give us a better understanding of the effect in the radiative properties. A typical procedure used to discern whether the ion levels are in LTE consists of evaluating the ratio between the population of each ion level obtained with the CRSS model and the one in LTE determined from the SB equations,

$$
b_{\zeta m}=\frac{p_{\zeta m \mathrm{CRSS}} / p_{\zeta 0 \mathrm{CRSS}}}{p_{\zeta m \mathrm{SB}} / p_{\zeta 0 \mathrm{SB}}},
$$

where $p_{\zeta m}$ and $p_{\zeta 0}$ are the fractional populations of the level $m$ and of the ground level of the ion $\zeta$, respectively. The values of $b_{\zeta m}$ are represented in the so-called $b$ plots [61] and there is one $b$ plot for each ion. In these plots, the $b$ values for the levels are represented against the ratio between the energies of the levels (with respect to the energy of the ground state) and the ionization potential of the ion considered,

$$
e_{m}=\frac{E_{\zeta, m}-E_{\zeta, 0}}{I_{\zeta}} .
$$

The explanation of the $b$ plots is simple: according to Eq. (30), values of the parameter $b$ near unity mean that the distribution of populations is near the LTE one. Furthermore, the ground state and low-lying excited levels have low values of $e_{m}$; as the value of $e_{m}$ increases the levels are more excited and the autoionizing levels are those with $e_{m}>1$. At $10 \mathrm{eV}$, we obtained that, for the density of matter of $10^{-2} \mathrm{~g} \mathrm{~cm}^{-3}$, the atomic configurations can be considered in LTE since the values of the $b$ parameter are very close to unity, see, for example, Fig. 4 (top), where we have represented the $b$ plot for the most abundant ion at this plasma condition. This fact implies that the monochromatic emissivities and opacities calculated using SB and CRSS models will be almost identical. Obviously, this result also holds true for the density of matter of $10^{-1} \mathrm{~g} \mathrm{~cm}^{-3}$ at that temperature. For the lowest density, where we had obtained that both the average ionization and the CSDs provided by both population kinetics model are very similar, the $b$ plot of the most abundant ion is shown in Fig. 4 (bottom). From the figure we observe that there is a large group 

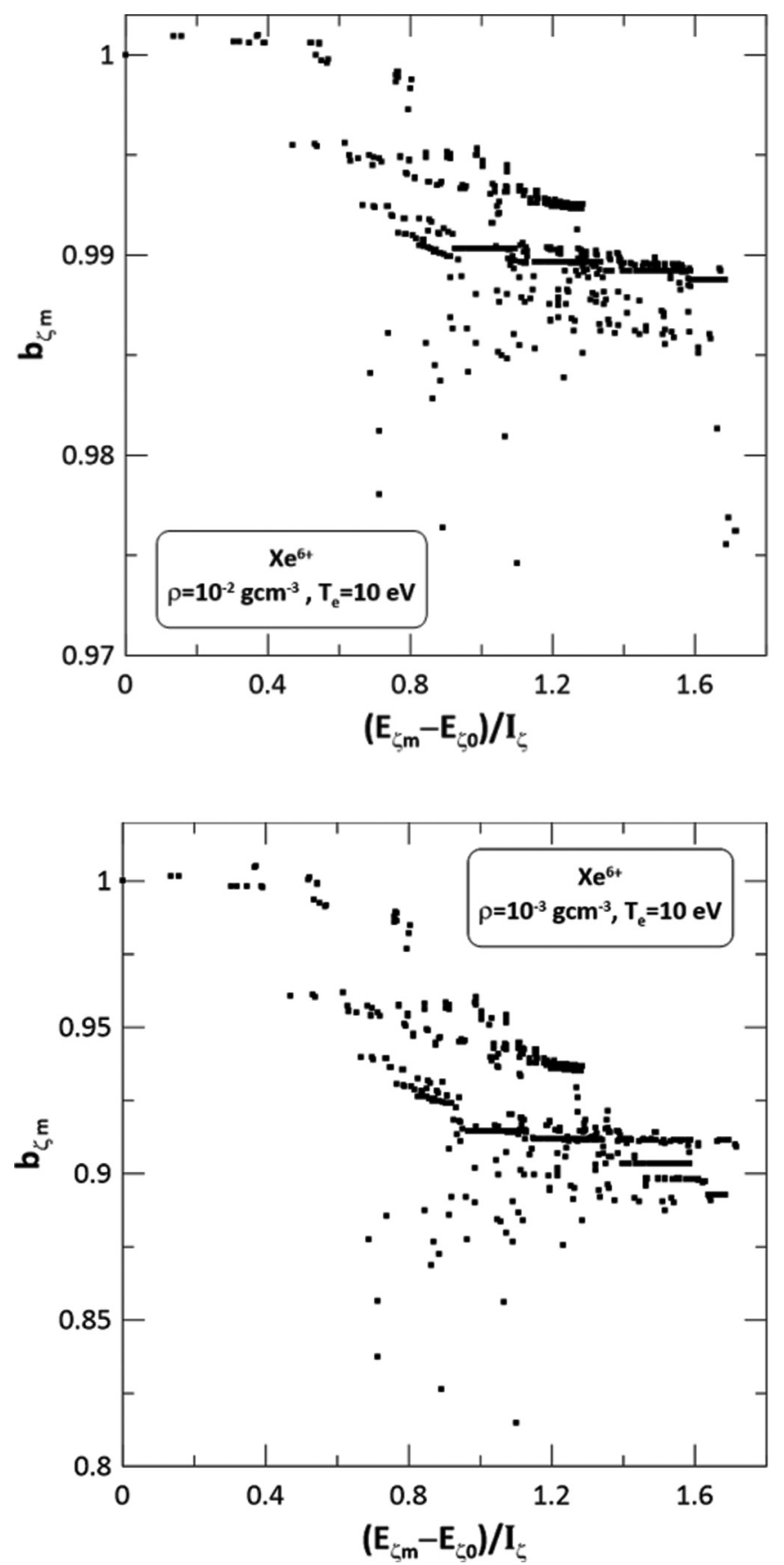

FIG. 4. $b$ Plots of the most abundant ion for the corresponding plasma conditions.

of atomic configurations whose values of the $b$ parameter range between 1.0 and 0.9, which indicates slight departures from the LTE. However, we have verified that these differences are not relevant enough to introduce significant deviations in the radiative properties calculated with both models.

As noted above, as the temperature increases, for a given density of matter, the departures from the LTE become larger. For this reason, we also analyzed the $b$ plots at $40 \mathrm{eV}$. From the analysis of the level populations, we have obtained that at this temperature for the density of matter of $10^{-1} \mathrm{~g} \mathrm{~cm}^{-3}$ the atomic configurations can be considered in LTE and there are no differences between the monochromatic emissivities

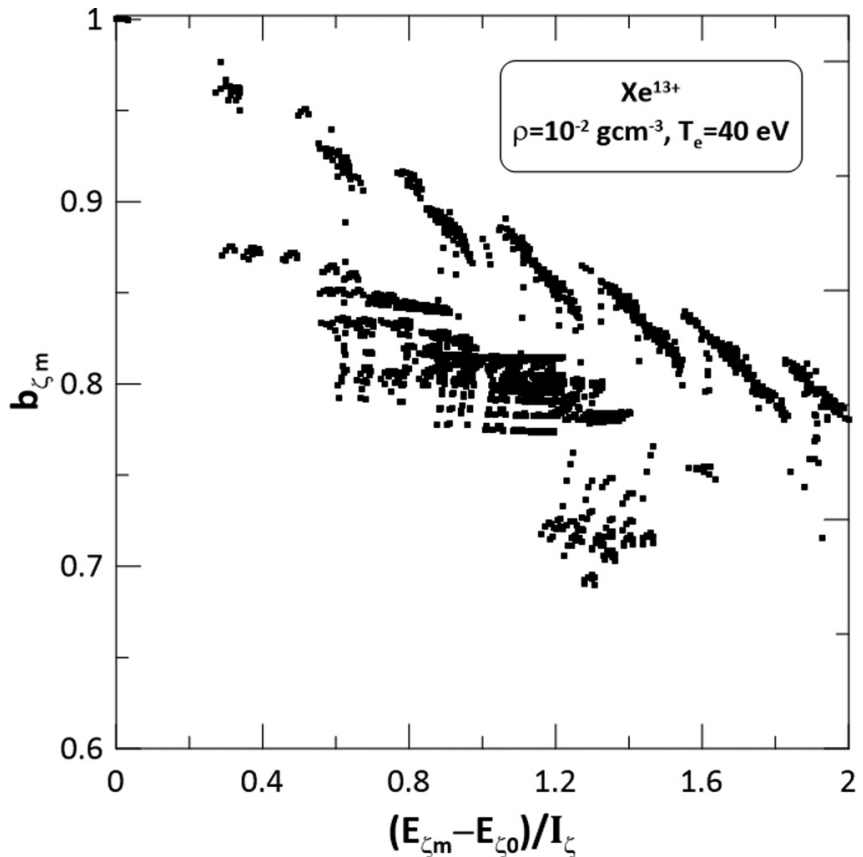

FIG. 5. $b$ Plot of the most abundant ion.

and opacities provided by SB and CRSS simulations. For the density of $10^{-2} \mathrm{~g} \mathrm{~cm}^{-3}$ and the same temperature we have represented in Fig. 5 the $b$ plot for the most relevant ion at this plasma condition. In this case, most of the atomic configurations have values of the $b$ parameter between 0.9 and 0.8 . There are also two smaller groups of configurations with values between 1 and 0.9 and lower than 0.8 , respectively. For this plasma condition we had obtained similar average ionizations and CSDs between LTE and NLTE simulations. However, from the $b$ plot we observe that many of the atomic configurations are in NLTE. This fact will introduce differences between the radiative properties calculated using both models. However, since the CSDs are quite similar, the ions involved in both simulations are the same. Therefore, the structures in the spectra are almost identical and there are only differences in the height of some peaks and the depth of the valleys, although they are not very important, as can be seen in Fig. 6. For the lowest density of matter, the levels are in NLTE, as maybe expected according to the results obtained for the average ionization and CSD. In this case, the average ionization in SB and CRSS simulations are 15.11 and 13.59 , respectively, and then the CSDs provided by both models present some differences (ions from $\mathrm{Xe}^{13+}$ to $\mathrm{Xe}^{17+}$ assuming LTE and from $\mathrm{Xe}^{12+}$ to $\mathrm{Xe}^{15+}$ in the NLTE simulation). This fact explains that there are some differences in the structures of the monochromatic opacities and emissivities spectra, as shown in Fig. 7. From the figure, more noticeable differences are detected as the photon energy increases. This could be due to the fact that the CSD of the SB simulation includes two more ionized ions, $\mathrm{Xe}^{16+}$ and $\mathrm{Xe}^{17+}$, that are not present in the NLTE simulation.

Since the Planck and Rosseland mean opacities and the RPL are obtained from the monochromatic opacities and emissivities, respectively, the differences between the SB and CRSS simulations will be related. Thus, from Fig. 8 we observe that the Planck and Rosseland mean opacities for the 


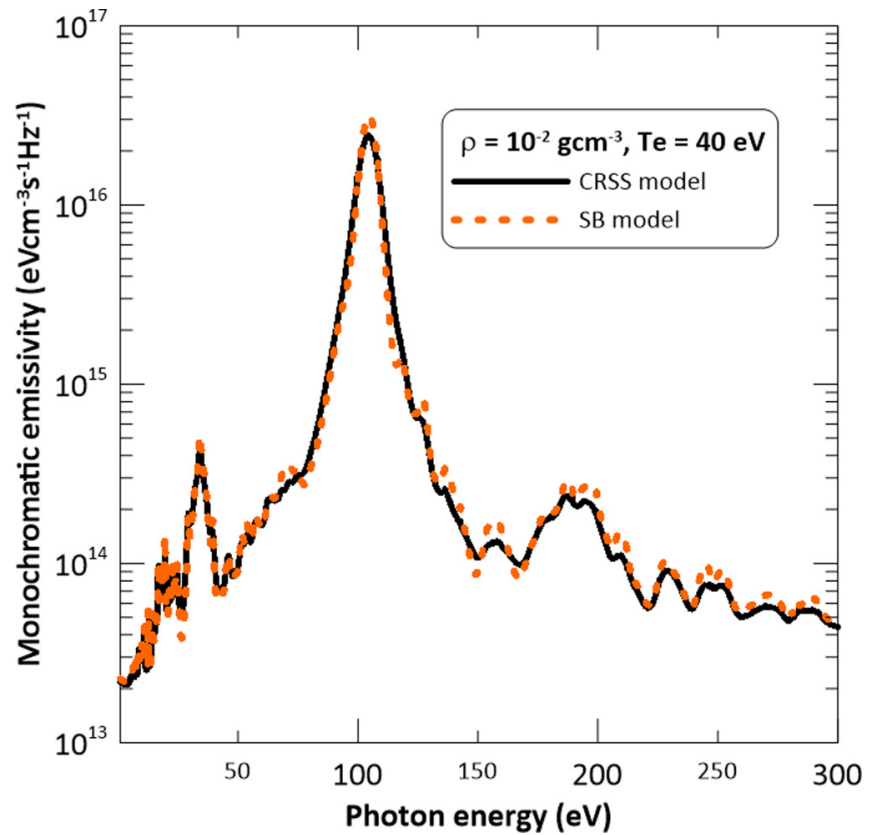

FIG. 6. (Color online) Comparison between the monochromatic emissivities calculated using SB and CRSS models for the plasma condition displayed in the legend.

two largest densities are almost identical. On the other hand, for the density of matter of $10^{-3} \mathrm{~g} \mathrm{~cm}^{-3}$ the differences are beginning to be detected for temperatures greater than $20 \mathrm{eV}$, and they reach their maximum values (around 30\% and 50\% for the Planck and Rosseland mean opacities, respectively) in the range of temperatures $40-50 \mathrm{eV}$, being the values of the mean opacities provided by the SB model lower than the ones obtained with the CRSS model. For the RPL we have obtained something similar: there are differences only for the lowest density, although in this case the values of the RPL obtained using SB model is greater than the ones given by the CRSS simulation, being the maximum values of the differences around $30 \%$.

\section{STUDY OF THE ATOMIC DESCRIPTION}

In this section we have made an analysis of the influence of the atomic description in the calculation of the radiative properties of xenon plasmas in the range of conditions under interest. In particular, we compare the DCA approach with the single-configuration DLA one and the DLA approach including configuration interaction $(\mathrm{CI})$ that we will denote as $\mathrm{DLA}+\mathrm{IC}$ in the following. We have detected that, for the range of temperatures under study, the most relevant contribution to both the absorption and emission spectra is the bound-bound. Therefore, for simplicity, we have opted to retain the DCA approach for the bound-free contribution.

A previous analysis of the general features of the wave functions of the orbitals and their overlapping gives us information about the relevance of the contribution of their transitions to the spectra. This is very useful for mediumor high- $Z$ elements, like xenon, as performing a complete CI calculation to obtain all necessary bound-bound oscillator strengths is intractable in these cases. Therefore, large-scale
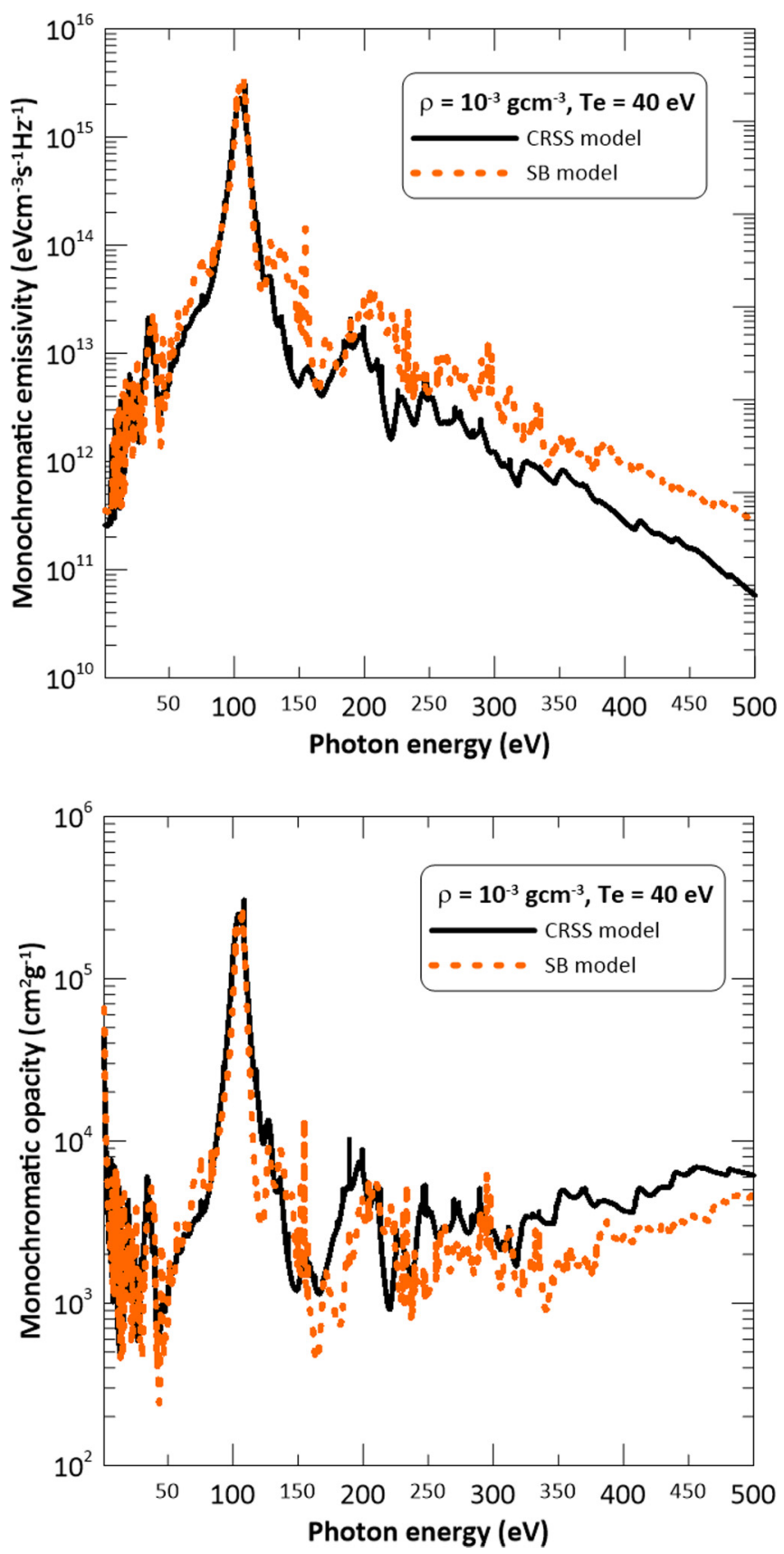

FIG. 7. (Color online) Comparison between the monochromatic emissivities and opacities calculated using SB and CRSS models for the plasma condition displayed in the legend.

CI calculations are only made for strong transitions while for the other less-relevant transitions a single-configuration DLA approach has been used. In Fig. 9 we show the square of the radial wave functions of the orbitals $4 d, 5 s, 5 p, 5 d, 6 s, 6 p, 6 d$ for $\mathrm{Xe}^{2+}$ and orbitals $4 s, 4 p, 4 d, 4 f, 5 s, 5 p, 5 d, 5 f$ for $\mathrm{Xe}^{9+}$. As noted above, the atomic structure was obtained in a relativistic context. However, as the difference between the relativistic radial wave functions of the same orbital angular momentum is small, we have represented in the figure the wave functions of nonrelativistic orbitals according to their statistical average.

For both ions, the wave functions of $5 s, 5 p$ and $5 p, 5 d$ have large overlaps, and, as a result, the oscillator strengths 

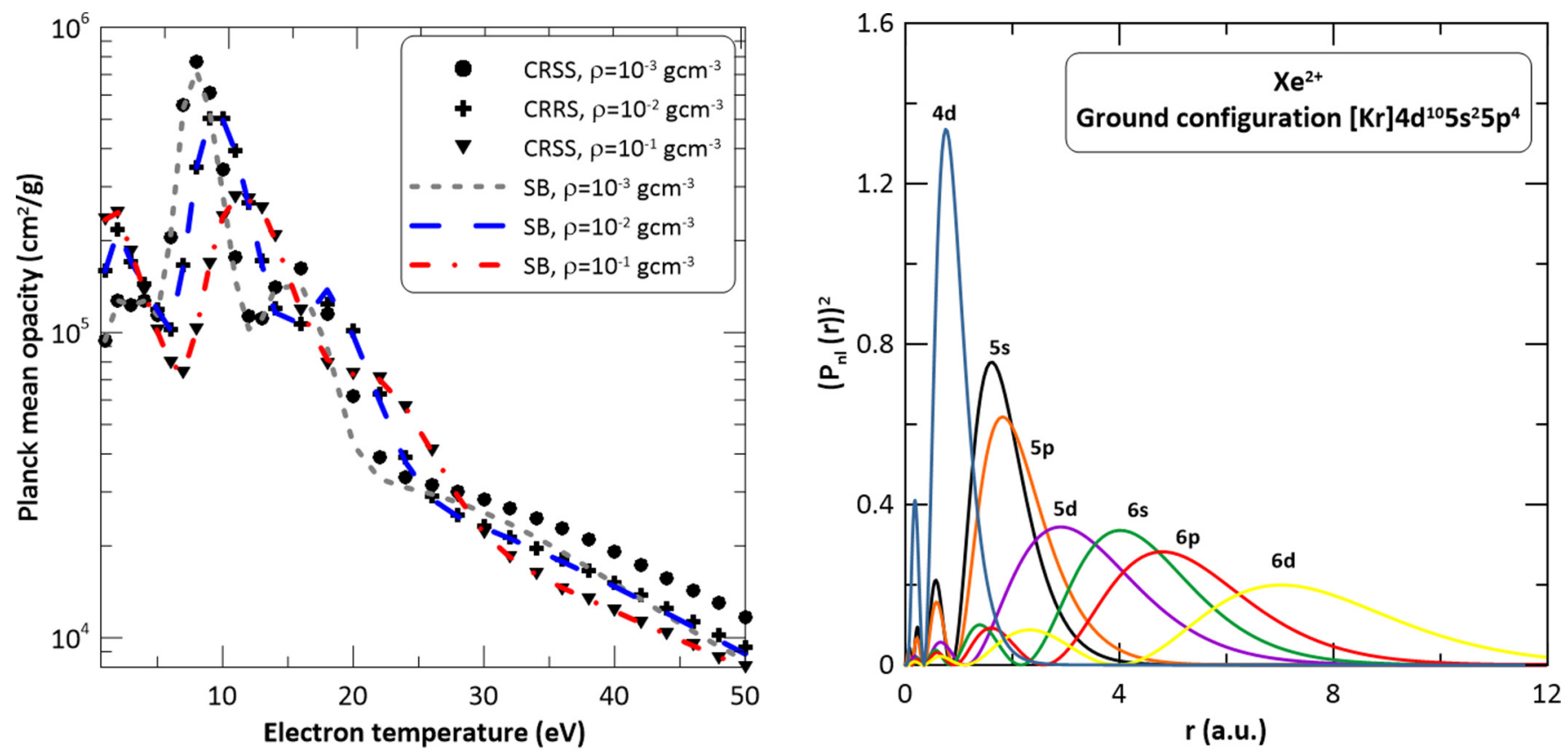

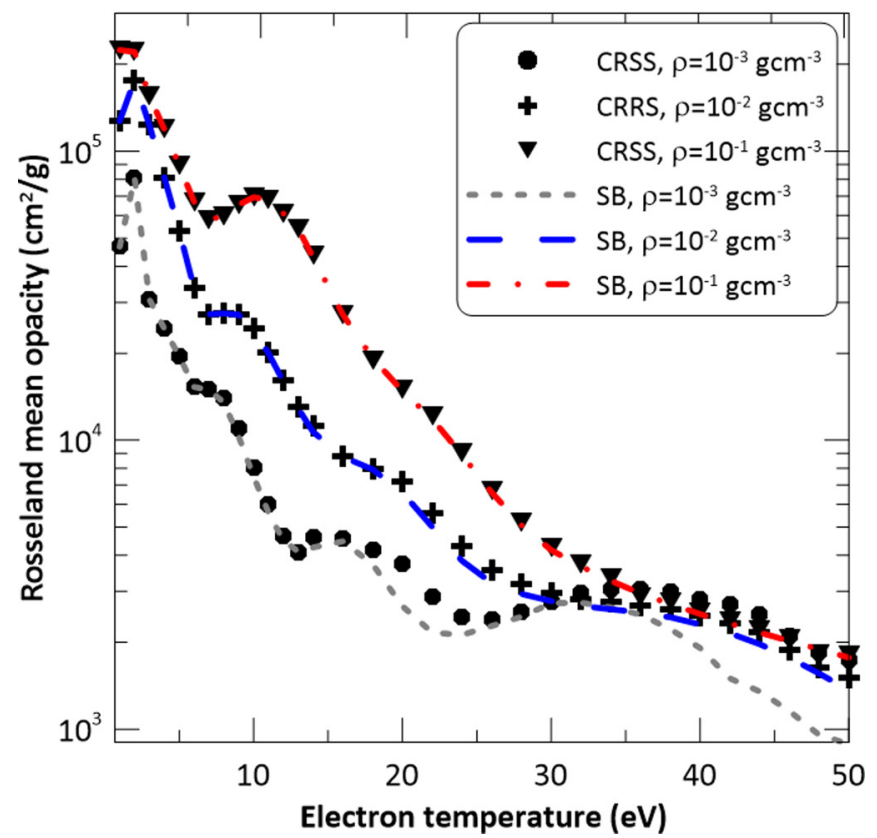

FIG. 8. (Color online) Comparison between the Planck (top) and Rosseland (bottom) mean opacities calculated using SB and CRSS models.

of the transitions between $5 s$ and $5 p$ and between $5 p$ and $5 d$ should be large. Besides, for $\mathrm{Xe}^{2+}, 5 p$ and $6 s$ orbitals are moderately overlapped. As the quantum number increases, $(n>6)$, the overlap of the wave functions with $5 s$ and $5 p$ orbitals decreases. For $\mathrm{Xe}^{9+}$ we observe a very large overlap between between $4 p$ and $4 d$ and then the oscillator strength should be large for the transition between these orbitals. More moderate is the overlapping between $4 d$ and $4 f$ and even more between $4 d$ and $5 p$, although they are still relevant.

Therefore, we have included in the DLA+CI model interaction among atomic configurations according to the

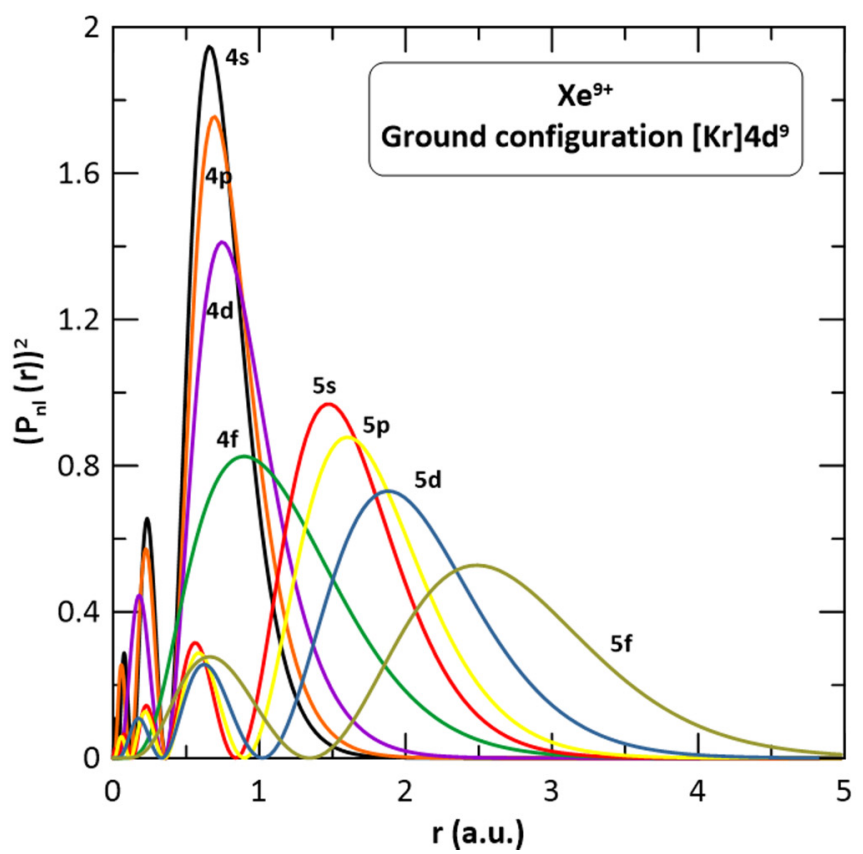

FIG. 9. (Color online) Overlap of the square of the radial wave functions of orbitals $4 d, 5 s, 5 p, 5 d, 6 s, 6 p, 6 d$ for $\mathrm{Xe}^{2+}$ and orbitals $4 s, 4 p, 4 d, 4 f, 5 s, 5 p, 5 d, 5 f$ for $\mathrm{Xe}^{9+}$.

results obtained from the previous study. We take $\mathrm{Xe}^{2+}$ and $\mathrm{Xe}^{9+}$ as examples of the scale of CIs included in the calculations carried out in this section. For the former ion fully CI has been considered for the following configurations: $5 s^{2} 5 p^{4}, 5 s^{1} 5 p^{5}, 5 p^{6}, 5 s^{2} 5 p^{3} 5 l, 5 s^{1} 5 p^{4} 5 l$ (with $l=$ $d, f), 5 s^{2} 5 p^{2} 5 d^{2}, 5 s^{2} 5 p^{2} 5 d^{1} 5 f^{1}, 5 s^{1} 5 p^{3} 5 d^{2}, 5 s^{1} 5 p^{3} 5 d^{1} 5 f^{1}$, $5 s^{2} 5 p^{3} 4 f^{1}, 5 s^{1} 5 p^{4} 4 f^{1}, 5 s^{2} 5 p^{3} 6 l^{\prime}$, and $5 s^{1} 5 p^{4} 6 l^{\prime}$ (with $l^{\prime}=$ $s-f)$. For $\mathrm{Xe}^{9+}$ the $\mathrm{CI}$ among the following configurations has been considered: $4 d^{9}, 4 p^{5} 4 d^{10}, 4 s^{1} 4 d^{10}, 4 d^{8} 4 f^{1}, 4 d^{7} 4^{f} 2$, $4 p^{5} 4 d^{9} 4 f^{1}$, and $4 d^{8} 5 l^{1}$ (with $l=s-d$ ). Since the simulations including $\mathrm{CI}$ increase the computing time considerably, 
we have only considered them in the most abundant ions at each plasma condition analyzed. For the other ions, a DLA description has been used but only in the single-configuration approach.

We have made a qualitative analysis of the influence of these atomic descriptions in the plasma properties. In particular, we have selected two electron temperatures in the range analyzed, 5 and $20 \mathrm{eV}$. This is due to the fact that these temperatures can be present in the kind of experiments that we analyze in the next section. For these two temperatures and in the range of matter densities of interest we can assume the plasma in LTE according to Sec. III. We have found a good agreement both for the average ionization and the CSDs provided by the three models. This result is expected since they were determined using the Saha equation whose dependence on the atomic description is not too strong. On the other hand, the differences are more noticeable for the monochromatic opacities and emissivities. In Fig. 10 we have represented the bound-bound contribution to the monochromatic opacities at these two temperatures. First, we can observe that the DCA approach predicts the gross profile of the DLA calculation. However, the DLA+CI simulation provides a fine structure of the opacity since it includes transitions that were not allowed in the DCA and single-configuration DLA approaches. This fact is more evident for the case of lowest temperature and density of matter represented, since the collisional and Doppler broadenings are lower than in the other two conditions and because of the overlapping among the lines. These differences in the monochromatic opacities, and in the emissivities as well, imply differences in the average radiative properties. Thus, for these plasma conditions, we have obtained relative differences in the Planck and Rosseland mean opacities between DCA and DLA+CI simulations that can reach $60 \%$ and $20 \%$, respectively. Between DLA and DLA $+\mathrm{CI}$ calculations the relative differences can reach $40 \%$ and $20 \%$, respectively. Therefore, it is clear that single-configuration DLA description does not introduce a significant improvement respect to the DCA approach. With respect to the RPL, in Fig. 11 we show the results obtained from its calculation under the three atomic descriptions. From the figure we can observe that, as happens in the mean opacities, the three models provide values of the RPL of the same order of magnitude but the differences between the DCA and DLA+CI simulations can reach $100 \%$ in the worst case. Between the DLA and DLA+CI simulations the maximum error is about $45 \%$.

Therefore, we have obtained that, for the average radiative properties, the three models provide values of the same order of magnitude but with relative errors that can reach $100 \%$ in the DCA approach and $40 \%$ in the DLA one with respect to the DLA+CI description. Therefore, it seems that the logical choice would be the DLA+CI description. However, we point out that both the generation of the atomic databases and the calculation of the radiative properties in this atomic description imply a considerable increase of the computational times. This is even more true in NLTE situations, where the set of rate equations must be solved and in which external radiation fields or plasma self-absorption could be involved. All these facts should be taken into account when we select an atomic description to perform, for example, radiative hydrodynamics simulations of the type of experiments considered in this work.
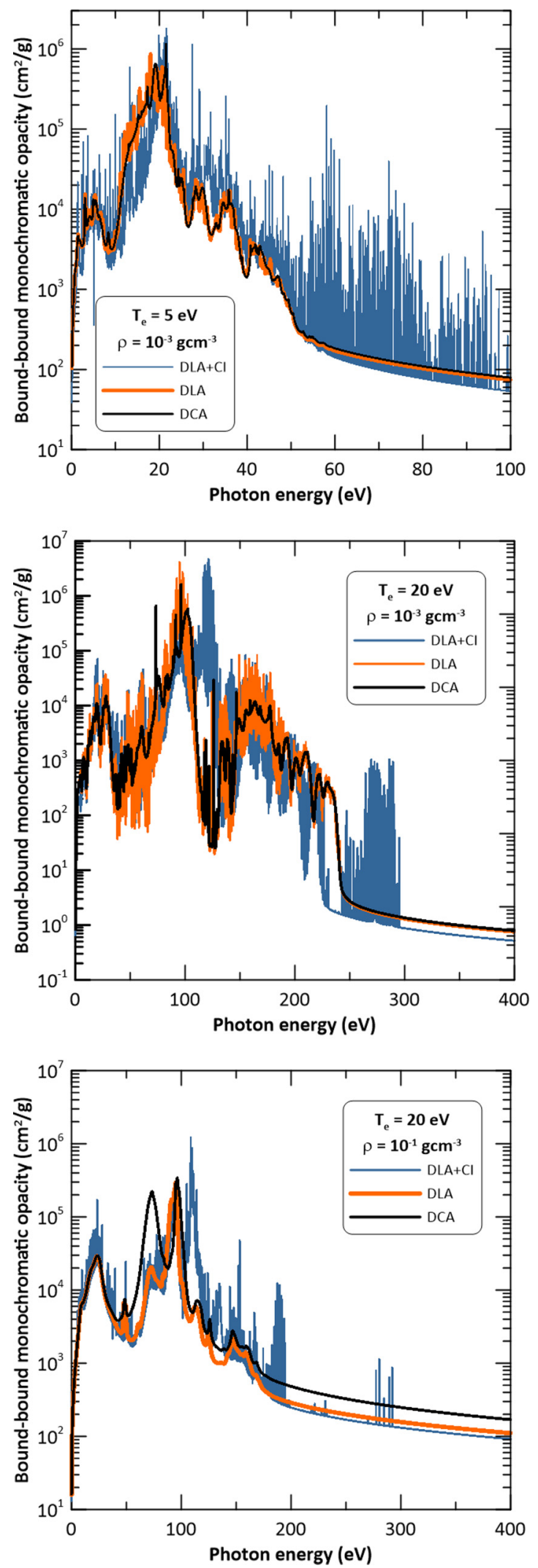

FIG. 10. (Color online) Comparison of the bound-bound contributions to the monochromatic opacities obtained with the different atomic models.

On the other hand, if we are interested in the analysis of either the absorption or emission spectra, then the DLA+CI 


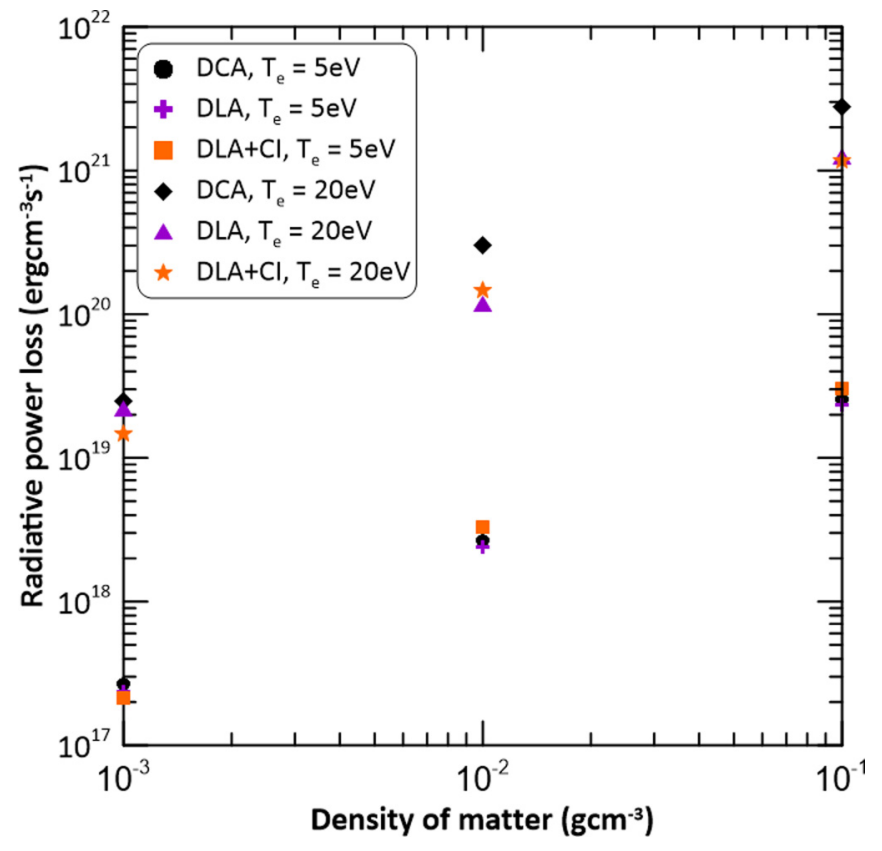

FIG. 11. (Color online) Comparison of the RPL obtained with the different atomic models for two electron temperatures as a function of the density of matter.

description should be used since the changes both in the position of the peaks and their number are significant. These changes might be more important in the NLTE regime since the rate equations are more sensitive to the atomic description than the SB equations and, therefore, even the average ionization and CSDs provided by the three models may differ.

\section{STUDY OF AN EXPERIMENTAL RADIATIVE SHOCK}

In this section we analyze the influence of the effect of the external radiation field and the plasma self-absorption on the microscopic properties of the radiative precursor of a radiative shock wave generated by a laser beam and propagating in xenon. Furthermore, we also study the possibility of the onset of thermal instabilities in the cooling layer behind the shock. For these purposes we have selected a particular experiment which is briefly described in the following.

\section{A. Description of the experimental setup}

The experiments were conducted at the Prague Asterix Laser System (PALS) and an extensive description of them can be found in Ref. [33]. We have limited ourselves here to point out some aspects relevant to our discussion. A beam from an iodine laser $(\lambda=1.315 \mu \mathrm{m})$ with a pulse duration of $0.3 \mathrm{~ns}$ drives a shock wave inside a target filled with a xenon gas at low pressure $\left(\approx 0.3\right.$ bar, i.e., $\left.\rho=1.5 \times 10^{-3} \mathrm{~g} \mathrm{~cm}^{-3}\right)$. The targets used for the shock generation consist of a miniaturized shock "tube," closed by a foil of gilded polystyrene ( $\mathrm{CH}$ and $\mathrm{Au}$ ). The beam is focused on this foil and the ablation of the polystyrene, due to the laser, propels by rocket effect the small section of the $\mathrm{Au}-\mathrm{CH}$ foil, which acts like a piston inside the shock tube. Then a radiative shock wave propagates in xenon with an average velocity around $50-60 \mathrm{~km} \mathrm{~s}^{-1}$ from 3 to $30 \mathrm{~ns}$ after the time of

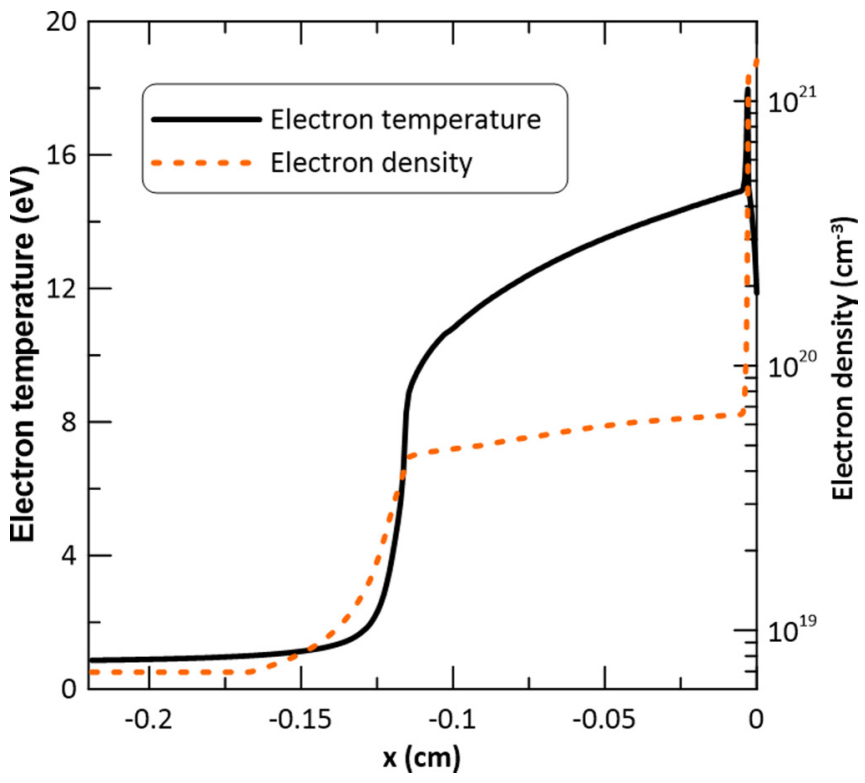

FIG. 12. (Color online) Snapshot of the plasma at $20 \mathrm{~ns}$ from MULTI 1D simulations [62].

the arrival of the laser beam on the $\mathrm{CH}$-Au piston. By means of a one-dimensional (1D) radiative hydrodynamical simulation using the MULTI code [62] the structure of the plasma was simulated. As an example, in Fig. 12 this is displayed at 20 ns. The shock is moving from the right to the left and only the simulation for the plasma of xenon is shown. The position of the shock front is located at $-0.003 \mathrm{~cm}$. At this position the electron density peaks at $10^{21} \mathrm{~cm}^{-2}$ (a density of matter around $4 \times 10^{-3} \mathrm{~g} \mathrm{~cm}^{-3}$, which is also the density of the shocked shell) and the electron temperature at $18 \mathrm{eV}$. The thickness of the shocked xenon layer was around $10 \mu \mathrm{m}$. Behind the shock front is the postshock region with a temperature between 12 and $17 \mathrm{eV}$ and a density of matter around $4 \times 10^{-3} \mathrm{~g} \mathrm{~cm}^{-3}$. Ahead of the front shock is observed the radiative precursor (between -0.22 and $-0.03 \mathrm{~cm}$, approximately). The $\mathrm{Au}-\mathrm{CH}$ piston is not represented in the figure.

\section{B. Plasma self-absorption in the radiative precursor}

We have analyzed the influence of the plasma selfabsorption in the calculation of the population of the atomic configurations of the radiative precursor. As said before, in ABAKO the bound-bound opacity effects are taken into account in population kinetics calculations by means of the escape factor formalism. For a given line transition $\zeta i \leftrightarrow \zeta j$, the escape factor $\Lambda_{j i}$ is introduced as an alternative way of writing the net rate of line emission. The escape factors enter in the calculations in two ways: first, in the atomic physics calculations of excited-state populations. As a result there is an effective reduction in the Einstein spontaneous emission coefficient $A_{\zeta j \rightarrow \zeta i}$, which is written as $\Lambda_{j i} A_{\zeta j \rightarrow \zeta i}$. Second, they appear in the determination of the total emergent line intensity. This modification circumvents the need to perform a simultaneous calculation of radiation transport and atomic physics. To compute the escape factors we have adopted the technique described in Ref. [42]. Thus, assuming a uniform 
distribution of emitting atoms and isotropic emission, for the three basic geometries - plane, cylindrical, and spherical - the escape factor $\Lambda_{j i}$ is written as

$$
\Lambda_{j i}=\int_{0}^{\infty} \phi_{i j}(v) \frac{1}{\tau_{i j}(v)} F\left[\tau_{i j}(v)\right] d v,
$$

where $\tau_{i j}(v)=\kappa_{\zeta i \rightarrow \zeta j}(v) L$ is the optical depth, where $L$ denotes the characteristic plasma dimension, i.e., slab width, cylinder, or sphere radius. Finally, $F\left(\tau_{i j}\right)$ is a functional of the optical depth whose particular form depends on the considered geometry. On the other hand, for each line transition, the escape factor depends implicitly on the populations of the lower, $N_{\zeta i}$, and upper levels, $N_{\zeta j}$, since they are required to compute the absorption coefficient [see Eq. (12)] and then the optical depth. Hence, in the case of optically thick plasmas, the system of rate equations must be solved iteratively until convergence is achieved within a prescribed tolerance. For our simulation we have employed the electron temperature profile provided by the hydrodynamic simulation at $20 \mathrm{~ns}$ (see Fig. 12) and, therefore, our calculations are a postprocessing of the hydrodynamic results. We have assumed that the radiative precursor has plane geometry and we have divided it into homogeneous layers characterized by average electron temperatures at the same density of matter of $1.5 \times 10^{-3} \mathrm{~g} \mathrm{~cm}^{-3}$ and they are listed in Table V. For the plane geometry, the functional $F\left(\tau_{i j}\right)$ in Eq. (32) is given by [37]

$$
F\left(\tau_{i j}\right)=\frac{1}{2}-E_{3}(\tau)
$$

where $E_{3}(\tau)$ is the third-order exponential integral.

We have analyzed the influence on the average ionization, the mean opacities, and the RPL. For the average ionization, we have obtained that the relative differences between both simulations are always lower than $2 \%$, and the discrepancies diminish as the layer is more distant from the shock front, i.e., as the electron temperature decreases (see Table V). This behavior is also obtained for the RPL, as Fig. 13 shows, but the differences are noticeably greater than for the average ionization, since the RPL is more sensitive to changes in the population of the atomic configurations, reaching maximum differences around $29 \%$. In both properties the self-absorption

TABLE V. Division in homogeneous layers of planar geometry of the radiative precursor. The density of matter is $1.5 \times 10^{-3} \mathrm{~g} \mathrm{~cm}^{-3}$ in all the layers.

\begin{tabular}{lcr}
\hline \hline Layer & $T_{e}(\mathrm{eV})$ & $D(\mu \mathrm{m})$ \\
\hline 1 & 15.73 & 5 \\
2 & 14.50 & 313 \\
3 & 13.54 & 260 \\
4 & 12.53 & 193 \\
5 & 11.53 & 140 \\
6 & 10.56 & 104 \\
7 & 9.55 & 45 \\
8 & 8.57 & 9 \\
9 & 6.08 & 9 \\
10 & 4.72 & 9 \\
11 & 3.54 & 19 \\
12 & 2.40 & 37 \\
\hline \hline
\end{tabular}

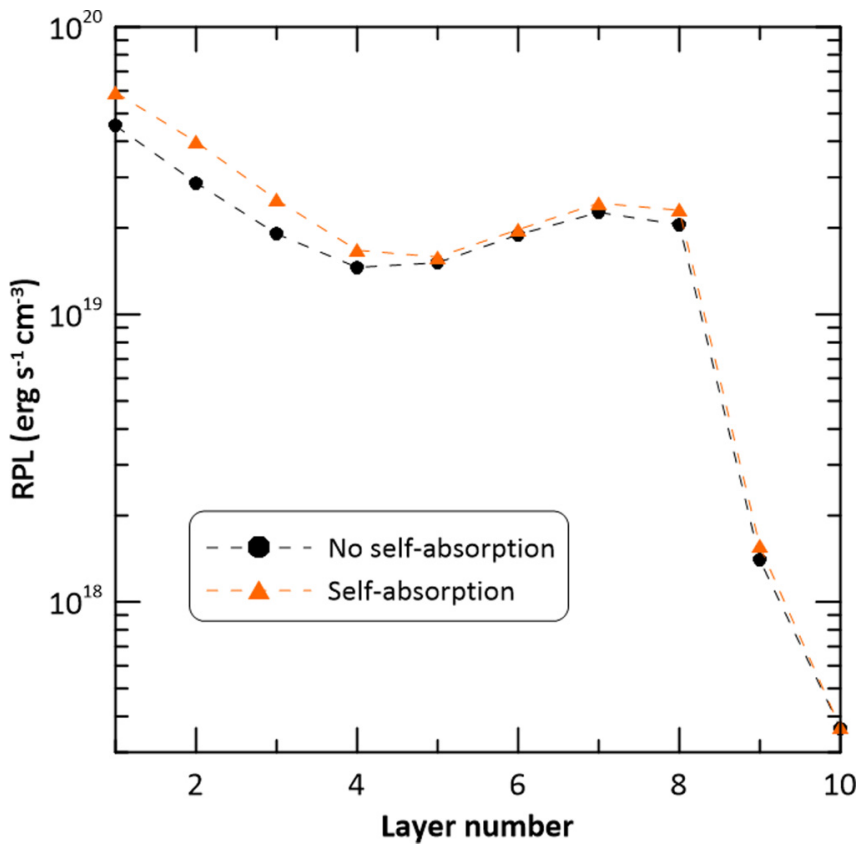

FIG. 13. (Color online) Comparison of the RPL calculated with or without including self-absorption in the population kinetics.

effect leads to an increase of them with respect to the simulation without opacity effects. As said before, the escape factor reduces the rate coefficient of the spontaneous decay process and then excited levels will be more populated, encouraging ionization by collisional processes.

With respect to the mean opacities the relative differences between both simulations are lower than the 5\% and $14 \%$ for Rosseland and Planck mean opacities, respectively. Rosseland mean opacity is determined by the depth of the valleys (and then on the line shapes) of the spectrum, whereas the Planck mean is determined mainly by the position and height of the peaks. Including self-absorption effects in the rate equations will change the populations of the atomic configurations and then the height of the peaks while the changes in the line shapes will be lower and this could explain why the differences are greater for the Planck mean opacity. Unlike for the average ionization and RPL, the relative differences between simulations with or without self-absorption effects do not present a regular trend with the temperature. Thus, we have obtained increases and decreases of these discrepancies as the temperature decreases, although the behavior of these fluctuations with the temperature is the same for both mean opacities. Unlike the radiative power loss, which is obtained from direct integration over frequency of the emissivity, mean opacities are computed by integrating the monochromatic opacities with their corresponding weighting functions [see Eqs. (24) and (25)], and therefore the result depends not only on the effect of the self-absorption on the monochromatic opacities but also on these functions, and this fact could explain the nonregular behavior with temperature of the relative differences of the mean opacities between both models.

In Fig. 14 we present the monochromatic opacity and emissivity for the layer number three of the radiative precursor. As we can see the differences are more relevant for the 

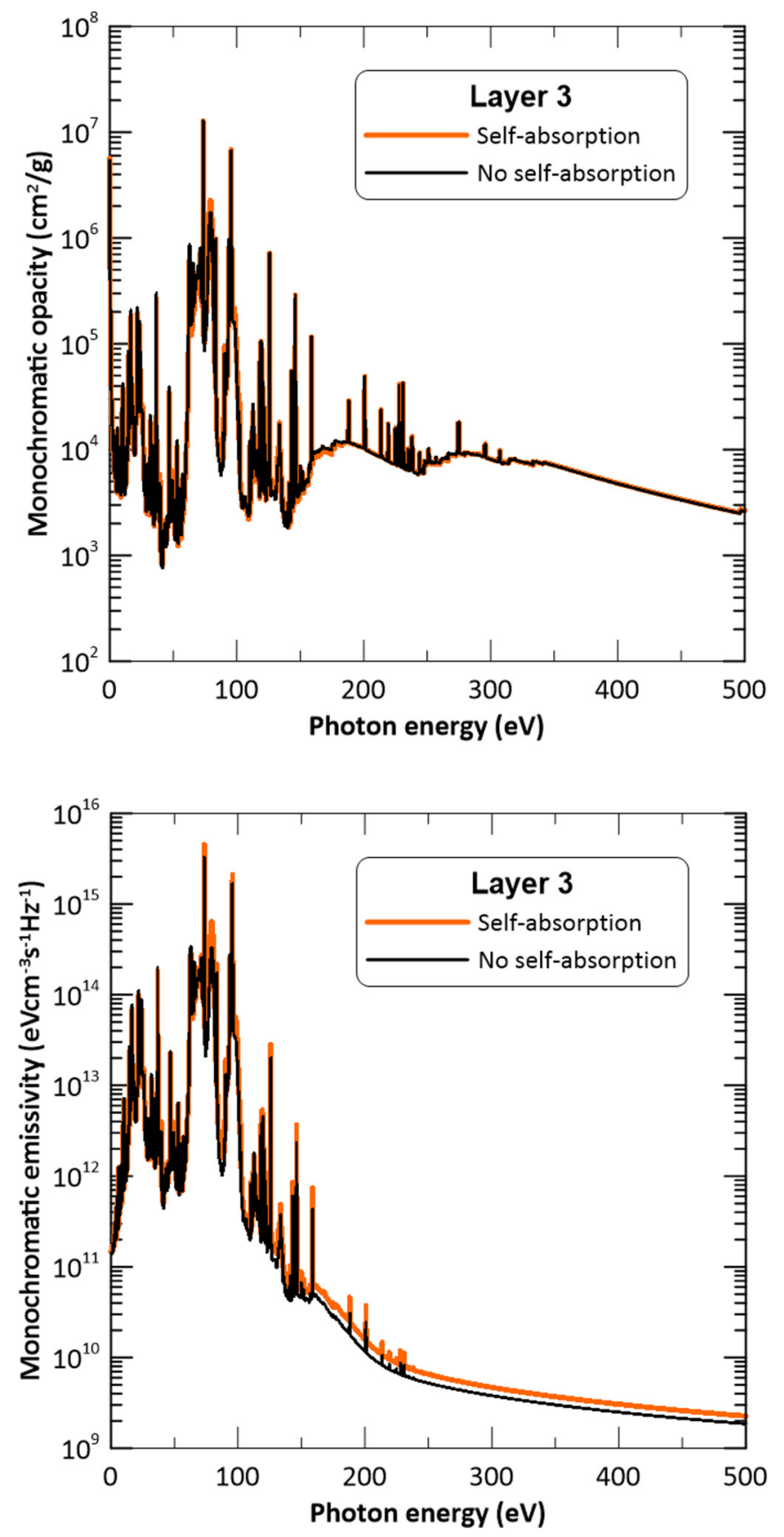

FIG. 14. (Color online) Comparison of the monochromatic opacity and emissivity calculated with or without including self-absorption in the population kinetic model for the layer number 3 of the radiative precursor.

emissivity than for the opacity. This fact implies that the self-absorption effects are more important in the excited configurations, since the opacity is more sensitive to the populations of the ground and first excited configurations than the emissivity.

We have not obtained differences between the radiative properties calculated with both models for the last five layers. The temperatures in these ones are always lower than $5 \mathrm{eV}$ (see Table V). In Sec. III we concluded that at this density of matter and for those temperatures the plasma can be considered in LTE. In this thermodynamic regime, collisional processes are the dominant ones and radiative processes are less relevant. This fact could explain why, for these layers, self-absorption effects are not noticeable. Finally, we would like to point out that the real differences between NLTE simulations with and without reabsorption effects might be lower than the ones presented before. This is due to the fact that the 1D planar model used in the escape factor formalism implemented in our code could overestimate them. Therefore, self-absorption would has no noticeable influence in the average ionization and mean opacities and for the RPL introduces changes for the first three layers and for layers. NLTE simulations including self-absorption in the layers significantly increase the computing time. Therefore, since the effects in the situation under analysis are not too relevant in the calculation of the mean radiative properties, they may not be considered in the population kinetics model. However, if we are interested in the comparison between experimental and simulated spectra or in spectroscopic diagnostics, which are more detailed analysis, these effects should be taken into account since as Fig. 14 shows there are differences in the intensity of some peaks of the spectra.

\section{Effects of the shock radiation in the radiative precursor}

We have employed the discretization of the radiative precursor in layers at $20 \mathrm{~ns}$ presented in the previous section to analyze the influence of external radiation fields in the collisional-radiative simulations of this region at that time. At each layer the incoming radiation will include the one emitted by the shock front and the radiation due to the self-emission of the previous layers, since we have not taken into account the radiation that comes from layers ahead of the one under consideration. The specific intensities are calculated from Eq. (6), assuming stationary situation for the radiation, along the beam in the propagation direction, which is given by

$$
I_{\nu}\left(\tau_{v}\right)=I_{\nu}(0) e^{-\tau_{\nu}}+\int_{0}^{\tau_{v}} S_{\nu}\left(t_{\nu}\right) e^{\left(\tau_{\nu}-t_{v}\right)} d t_{\nu},
$$

where $\tau_{v}$ is now the monochromatic optical thickness measured along the beam

$$
\tau_{\nu}(r)=\int_{0}^{r} \kappa_{s} d s .
$$

To compute the outgoing intensity from the shock shell we assumed that the plasma was homogeneous in which the source function did not change with the position, with planar geometry of thickness $D_{S}$ calculated as the full width at half maximum of the shock shell $(10 \mu \mathrm{m})$. Then, assuming $I_{\nu}(0)$ in Eq. (34), the outgoing intensity would be given by

$$
I_{\nu, S}\left(D_{S}\right)=S_{\nu}\left[1-e^{-\tau_{\nu}\left(D_{S}\right)}\right] .
$$

Assuming that a layer $j$ has a length denoted by $D_{j}$, the specific intensity of the incoming radiation to that layer is given by

$$
I_{v, j}=I_{v, j-1}\left(D_{j-1}\right)+S_{v, j-1}\left[1-e^{-\tau_{v, j-1}\left(D_{j-1}\right)}\right],
$$

and this radiation will be responsible for the radiative-driven processes in that layer. For $j=1$, i.e., the first layer, $I_{v, j}=$ $I_{\nu, S}\left(D_{S}\right)$. 


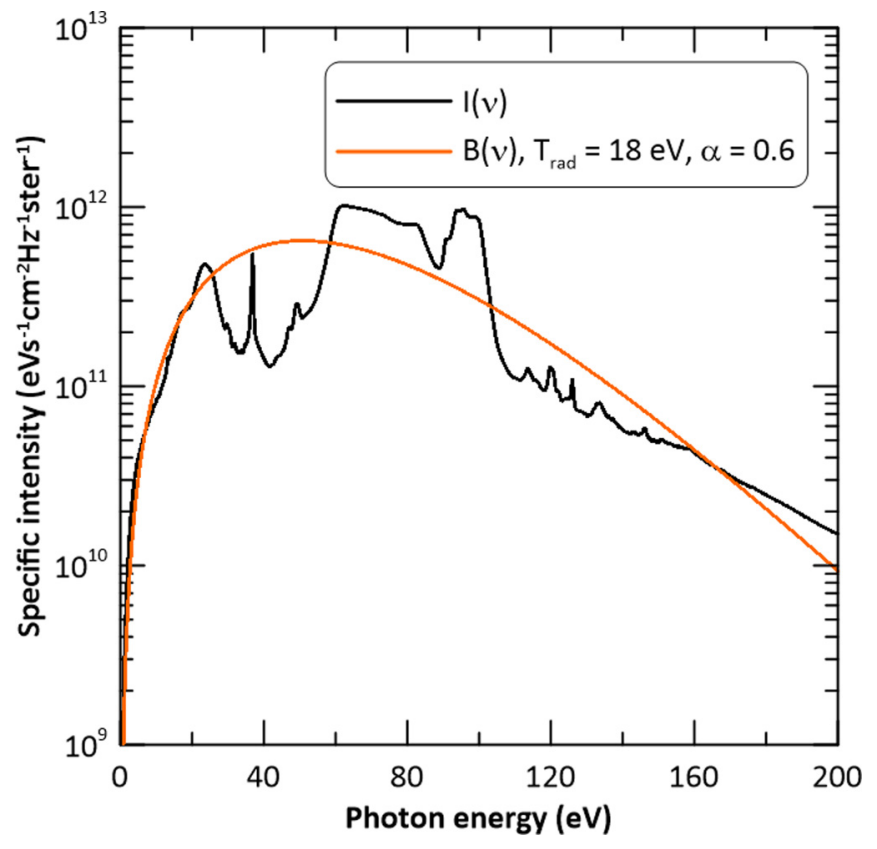

FIG. 15. (Color online) Outgoing specific intensity from the shock front along the beam in the propagation direction at $20 \mathrm{~ns}$ $\left(n_{e}=10^{21} \mathrm{~cm}^{-3}\right.$ and $T_{e}=18 \mathrm{eV}$ ) and Planck function with a radiation temperature of $18 \mathrm{eV}$ and a dilution factor $\alpha=0.6$.

First, we have studied the outgoing intensity of the shock front at $20 \mathrm{~ns}$. For this purpose we have assumed the shocked shell to be a homogeneous plasma with planar geometry with a width of $10 \mu \mathrm{m}$. We have taken as electron density and temperature the ones provided by the hydrodynamic simulation at the shock front at this time, i.e., $10^{21} \mathrm{~cm}^{-3}$ and $18 \mathrm{eV}$, respectively. Then the specific intensity along the beam in the propagation direction will be given by Eq. (36). In Fig. 15 we have represented this property and the Planck function at $18 \mathrm{eV}$ and with a dilution factor, $\alpha=0.6$, since, as noted above, in the radiative-driven processes in our NLTE kinetic model the radiation field is modeled by a Planck function. Qualitatively, the agreement between both curves is acceptable in the range of photon energies represented. Therefore, we will approximate the specific intensity by that diluted Planck function. In Fig. 16 we have represented the monochromatic opacities calculated at the density of matter of the radiative precursor and at two extreme temperatures, 15 and $2 \mathrm{eV}$. At temperatures close to $15 \mathrm{eV}$, i.e., the first layers of the radiative precursor, the absorption mainly occurs in the range $60-120 \mathrm{eV}$ as the figure shows. As the temperature decreases the ionization also does, and the range of photon energies in which the absorption is more significant is shifted toward lower energies. Thus, at the temperature of $2 \mathrm{eV}$, the range is about 15-70 eV and the absorption for energies larger than $100 \mathrm{eV}$ is considerably lower. Therefore, the range of photon energies in which the radiative precursor will have a larger absorption will be between 10 and $150 \mathrm{eV}$.

In Fig. 17 we have represented the intensity of the incoming radiation to the layer, which is modeled by a diluted Planck function and denoted as $B_{v, \text { in }}(0)$ in the figure and this intensity at the end of the layer, i.e., propagated through the layer of width $D$, is denoted as $B_{v \text {,in }}(D)$. Furthermore, the figure also
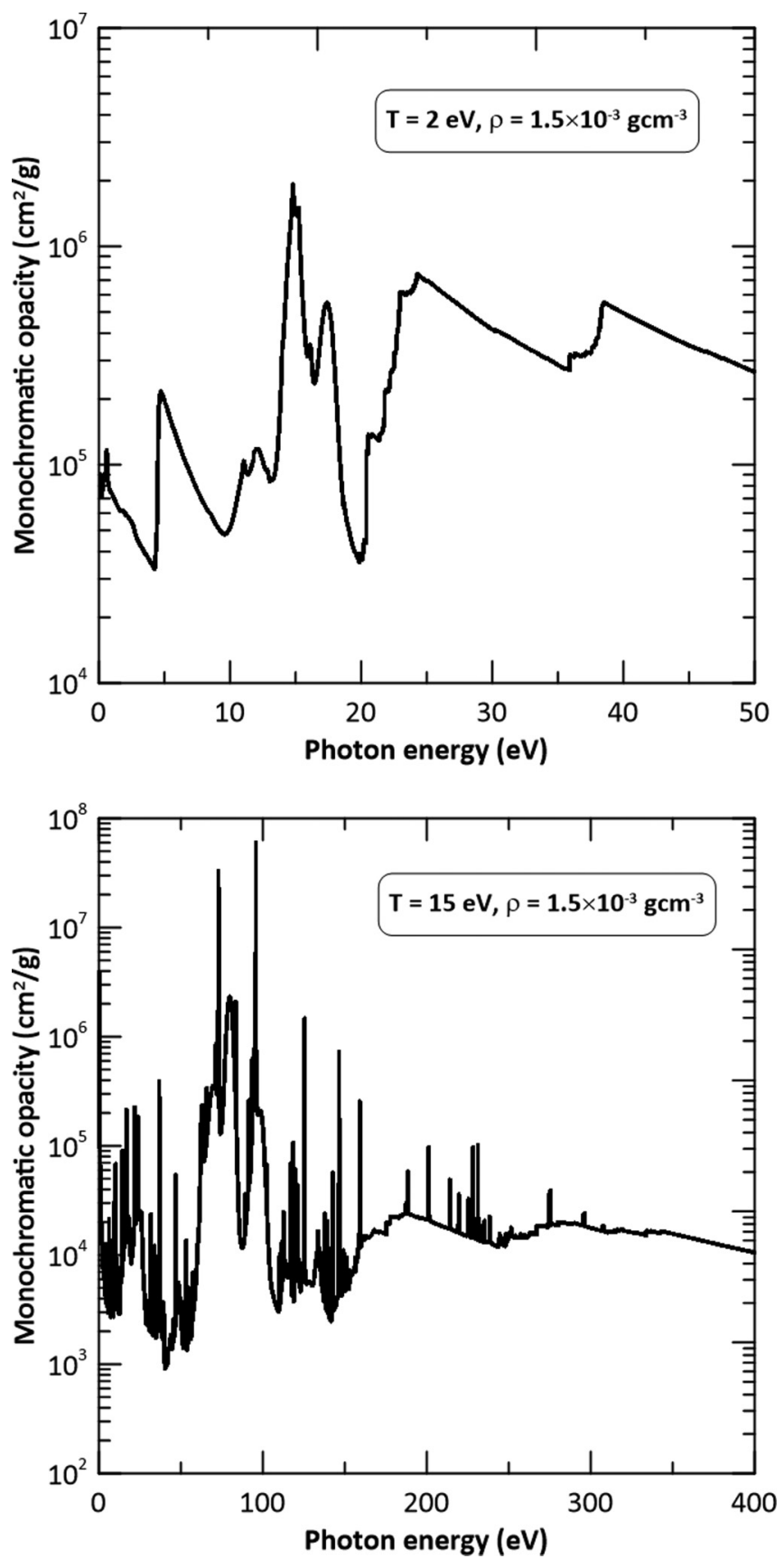

FIG. 16. Monochromatic opacities at two extreme temperatures of the radiative precursor.

shows the intensity of the radiation emitted by the layer itself, i.e., plasma self-emission, at the end of the layer $\left[I_{v}(D)\right]$, the layer outgoing intensity $\left[B_{v \text {,in }}(D)+I_{v}(D)\right]$, and the diluted Planck function that is used to approximate it, $B_{v, \text { out }}(D)$, that will be the incoming intensity to the following layer. All of them are represented for two layers, 2 and 5, of the radiative precursor, the former very near to the shock front and the latter somewhat further away. We have obtained that the contribution of the plasma self-emission radiation is very relevant and has to be taken into account. In the figures we can observe that, for example, in the range of photon energies $60-80 \mathrm{eV}$ there is a very strong absorption in the layer which is partially 

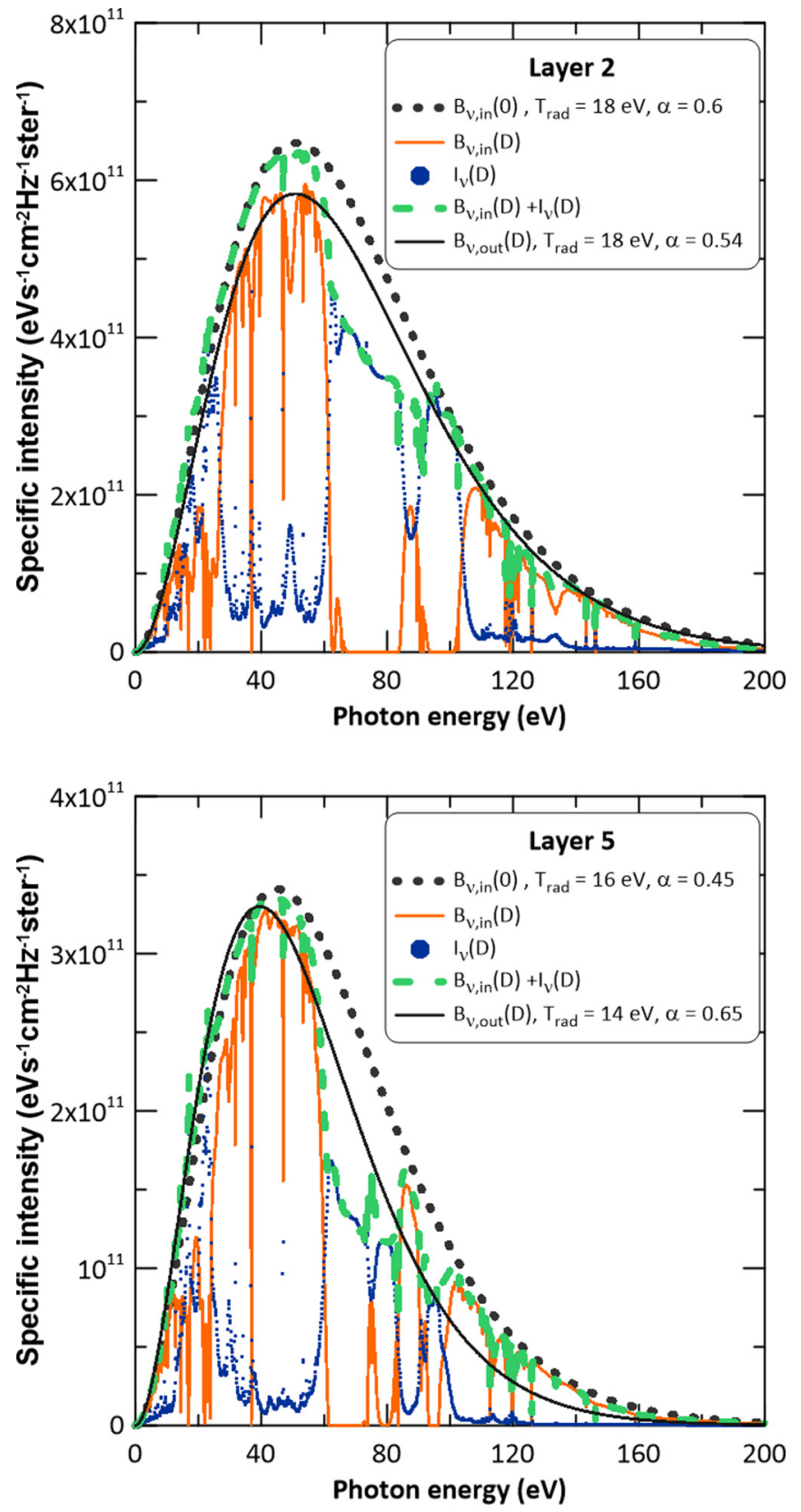

FIG. 17. (Color online) Incoming and outgoing intensities of layers 2 and 5 of the radiative precursor. Both intensities are approached by diluted Planck functions.

compensated by the radiation emitted by the layer itself. If this one was not included in the outgoing intensity, then the effect of the radiation in the following layer would be reduced. On the other hand, the radiation due to the plasma self-emission decreases as the layer is further away from the shock front, since the temperature decreases with the distance as Fig. 18 shows.

With respect to the influence of the radiation field on the microscopic properties, in Fig. 19 we have represented a comparison between the average ionization of the layers obtained including or not the external radiation field. As expected, the average ionization is greater when the radiation is included in the simulation, the relative differences always

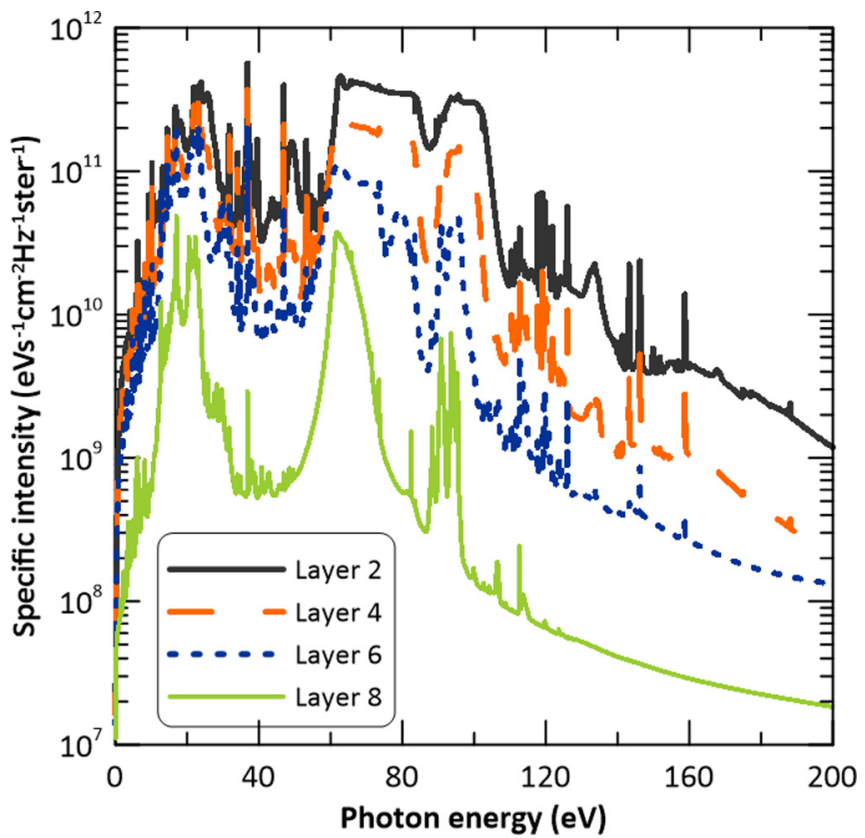

FIG. 18. (Color online) Specific intensities of the outgoing selfemission radiation of some layers of the radiative precursor.

being lower than $3 \%$. We observe that for the layer number 9 both simulations provide very close values. These two facts combined could contribute to this result. First, the radiation of the self-emission of the previous layer may be not too relevant. Second, the electron temperature of the plasma in this layer is about $6 \mathrm{eV}$ (see Table V). According to the discussion presented in Sec. III, for this density and this temperature the

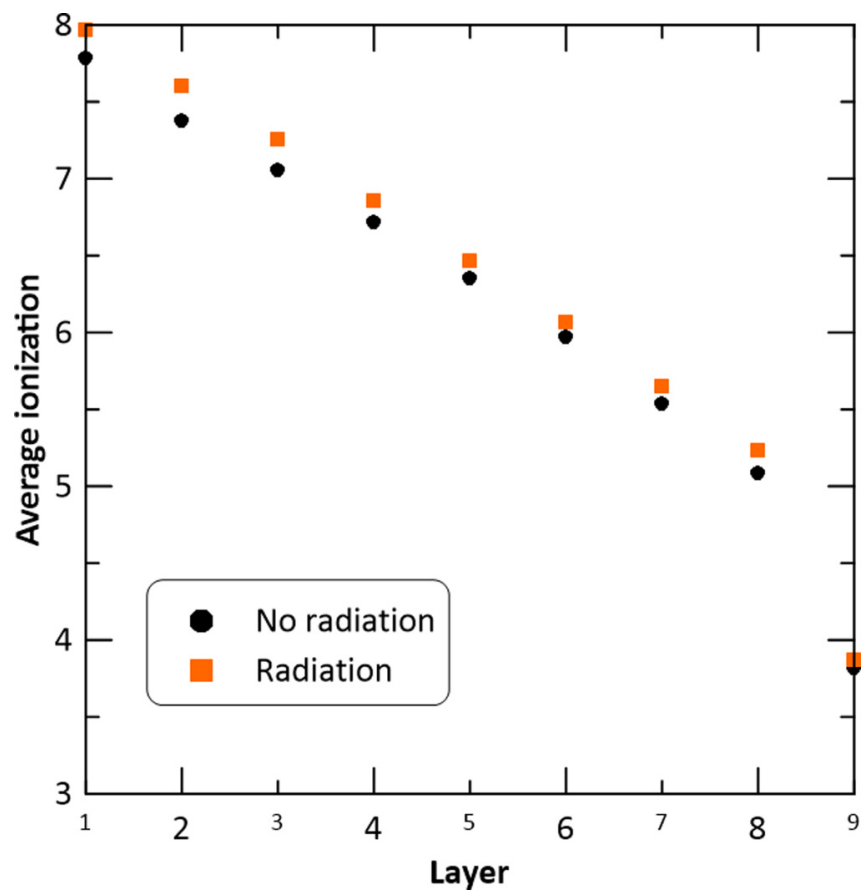

FIG. 19. (Color online) Comparison between the average ionization of the layers obtained including or not the external radiation field. 


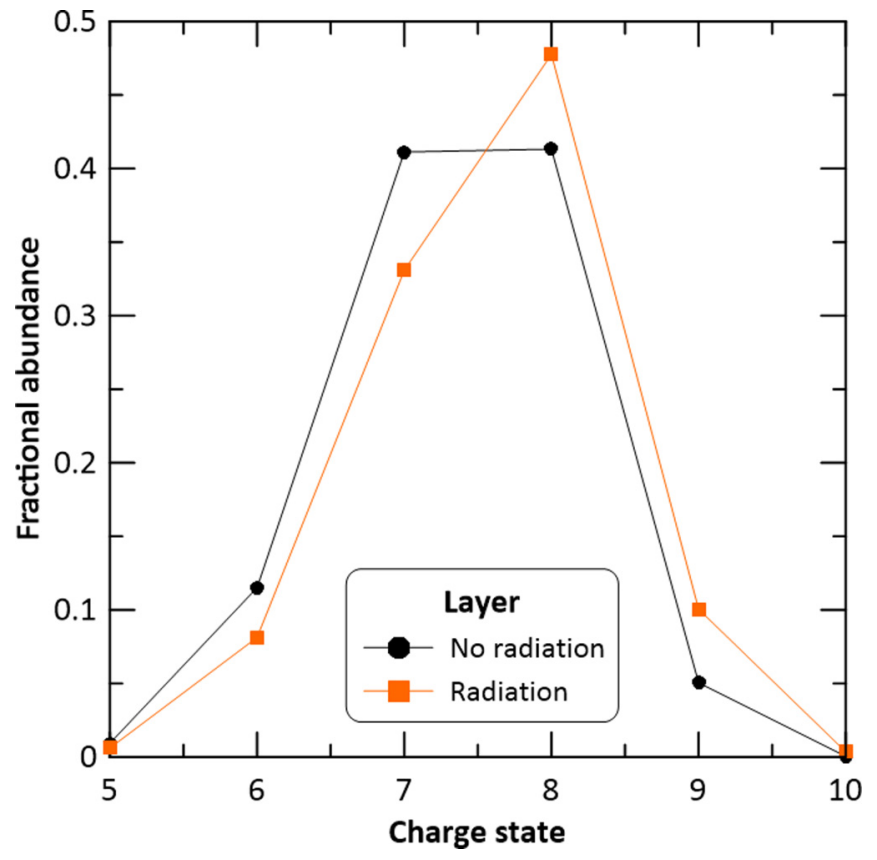

FIG. 20. (Color online) Comparison between the CSDs of layer 2 obtained including or not the external radiation field.

average ionization provided by NLTE was the same as the one of a LTE simulation and this means that collisional processes are more relevant than radiative ones. Therefore, if the external radiation is not too strong its effect will be small, although in this layer some slight differences are still observed in the CSD and the radiative properties. These differences continue to decrease in the following layers.

In Fig. 20 we have represented the comparison between the CSDs calculated including or not the external radiation field for layer 2. From the figure we observe that the radiation field appreciably modifies the fractional abundance of the charge states. Obviously, these changes will affect the monochromatic opacities and emissivities as Fig. 21 illustrates. In the figure we observe that since the ions that contribute to the spectra are the same in both simulations, the spectra present similar structures. However, the increase of the ionization due to the induced radiative processes produces an increase of the heights of the peaks associated to transitions in the range of photon energies higher than $80 \mathrm{eV}$ and also in the free-bound emission, whereas the contribution of the transitions of lower energies is reduced. Finally, the variations in the monochromatic radiative properties will affect the mean radiative properties. Thus, for the Planck and Rosseland mean opacities we have obtained maximum relative differences between both simulations lower than $20 \%$, whereas for the RPL these differences can be larger, about $50 \%$. We have also observed that although both simulations provide similar average ionizations and CSDs there are differences in the mean opacities and in the RPLs. In particular, for the simulation including the radiative induced processes the mean opacities are lower and the RPLs are larger than the ones obtained when the radiation is not considered. In this case, for each ion charge state we have observed that the populations of the excited configurations obtained when the external radiation field is included are larger than

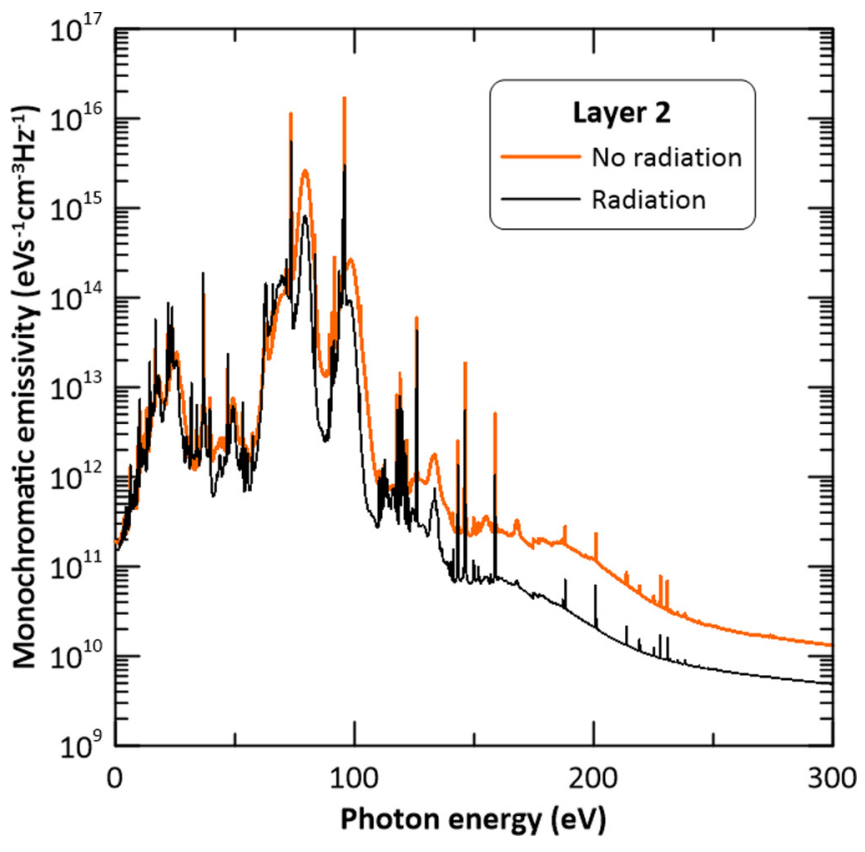

FIG. 21. (Color online) Comparison between the monochromatic emissivities of layer 2 obtained including or not the external radiation field.

when the radiation is not considered. As a consequence, the opposite occurs for the populations of the ground and first excited configurations. This could be due to the fact that the photoexcitation rate is greater than the photodeexcitation one. These facts may explain the results obtained for the monochromatic opacity and emissivity.

Therefore, from this qualitative analysis it seems that the radiation field due to both the shock front and the plasma self-emission affects the calculation of the plasma-level populations and the radiative properties in the precursor overall in the regions nearest to the shock front. Obviously, a precise calculation would imply a more appropriate treatment of the radiation field than the qualitative one made in this work.

\section{Radiative cooling time}

The radiative cooling time, $t_{\text {cool }}$, is given (in s) by

$$
t_{\mathrm{cool}}=2.42 \times 10^{-12} \frac{(\bar{Z}+1) n_{\mathrm{ion}} T_{e}}{\nabla \cdot \vec{F}_{\mathrm{rad}}},
$$

where $\vec{F}_{\text {rad }}$ is the radiative flux and its divergence (given in erg $\mathrm{cm}^{-3} \mathrm{~s}^{-1}$ in the previous equation), and if the radiation does not depend explicitly on time, it is given by

$$
\nabla \cdot \vec{F}_{\mathrm{rad}}=4 \pi \int_{0}^{\infty} j(v) d v-4 \pi \int_{0}^{\infty} \kappa(v) J(v) d \nu,
$$

where $J(v)$ is the mean spectral intensity. For simplicity, we have omitted in the previous equation the dependence of the radiative properties on time, position, and propagation direction, although they depend on them. Radiative cooling time is an interesting parameter since it allows us to determine whether the shock is radiative. With that purpose we have to compare $t_{\text {cool }}$ with the convective transport time $\left(t_{\text {conv }}=h / s\right.$ with $s$ the speed of the sound in the medium and $h$ hydrodynamic 
characteristic size). When $t_{\text {cool }}<<t_{\text {conv }}$, then the shock can be considered radiative. Furthermore, the radiative cooling time is also useful in order to classify the thermal cooling instabilities as we will show in the next section. For these reasons, we have made a previous analysis of the cooling time for the plasma conditions of interest in the experiment under analysis. First, we have studied the effect of the opacity on the calculation of the divergence of the radiative flux. With that purpose we have approached the mean spectral intensity in Eq. (39) by a diluted Planck function of a certain radiation temperature. We have obtained that the relevance of this term is considerably lower than the other one in the calculation of the divergence of the radiative flux and, therefore, this one can be approximated to the RPL. According to this result, we computed the radiative cooling time which is represented in Fig. 22 as a function of the temperature for three matter densities, $10^{-3}, 10^{-1}$, and $4 \times 10^{-2} \mathrm{~g} \mathrm{~cm}^{-3}$, where the latter is approximately the density of matter of the cooling layer. The electron temperature in the cooling layer in this experiment is between 17 and $12 \mathrm{eV}$. From the figure we observe that the cooling time shows a slight dependence with density of matter. With respect to the temperature, we detect an abrupt diminution up to temperatures around $10 \mathrm{eV}$, which means that the RPL increases considerably with the temperature, and for higher temperatures the cooling time presents a slight dependence with temperature, being almost constant for temperatures between 30 and $50 \mathrm{eV}$, and these results could be related to the behavior of the average ionization. This one includes a fast increase of $1-10 \mathrm{eV}$ from almost 0 to 4 (or 6 in the lowest-density case), see Fig. 2, removing electrons from the $5 p$ shell. On the contrary, in the range 30 and $50 \mathrm{eV}$ the increase is more moderate (from 9.88 to 13.63 for a density of $4 \times 10^{-2} \mathrm{~g} \mathrm{~cm}^{-3}$, for example) since the electrons belonging to the $4 d$ shell are now involved and higher temperatures are required to remove them.

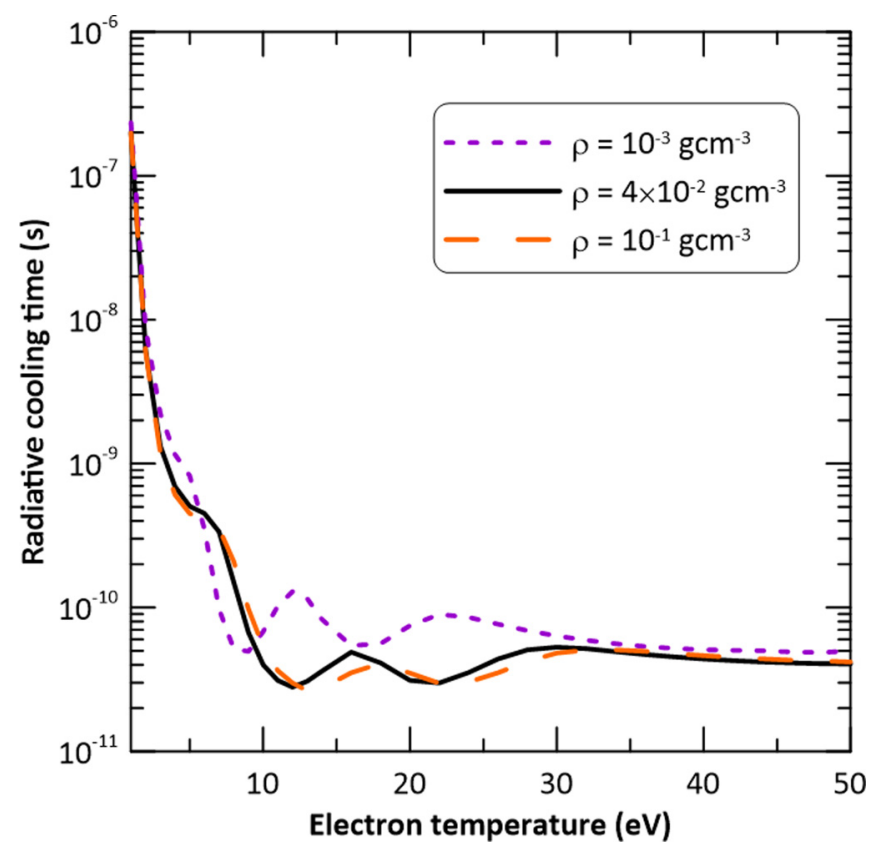

FIG. 22. (Color online) Radiative cooling time as a function of the temperature at three matter densities.
For the temperatures corresponding to the cooling layer $(15-17 \mathrm{eV})$ the cooling time is always around $3 \times 10^{-11} \mathrm{~s}$. On the other hand, the convective transport time is of the order of 1-10 ns, which is two orders of magnitude greater than the radiative cooling time, which corroborates the classification of the shock as radiative. We showed in Sec. IV that although the RPL provided by the DLA+CI simulations can differ from the ones obtained in the DCA simulations, they were of the same order of magnitude, and, therefore, this result is also maintained in the DLA+CI atomic description.

\section{E. Thermal instabilities in the cooling layer}

Thermal instabilities describe those instabilities that occur due to a balance between heating and cooling rates and they have been a topic of high interest in astrophysics over the past four decades [63-69]. This is due to the fact that they could be related to the formation of many astrophysical objects (for instance, the stars from the interstellar clouds). Nowadays, since scaled version of astrophysical phenomena can be recreated in the laboratory, interest in the study of thermal instabilities has been heightened.

Field [63] established the criteria for thermal instability, focusing particularly on the thermodynamics of the gas, assuming the unperturbed initial states as uniform and isothermal with no preexisting velocity fields. Thereafter, Hunter [64] made the first attempt to generalize the criteria for thermal instability in a nonstationary medium in the limit of short-wavelength perturbations. Shchenikov [65] obtained criteria for the thermal instability for nonstationary mediums both for short-wave and long-wave perturbations. For our purpose, which is the analysis of the possibility that thermal instabilities can occur in the cooling region behind the shock, we consider it to be more appropriate that the medium is nonstationary. Therefore, we have followed the method developed by Shchenikov [65]. A full description of the procedure can be found in that reference. Here we have limited ourselves a brief explanation.

The thermal energy equation, taking into account volume losses for a nonstationary medium, is given by

$$
\frac{d U}{d t}=\frac{P}{\rho^{2}} \frac{d \rho}{d t}-L
$$

where $U$ is the internal energy of the gas per gram, $U=\frac{p}{(\gamma-1) \rho}$, $P$ is its pressure, $\gamma$ denotes the adiabatic index, and $L$ is the rate of heat loss (the cooling rate minus the heating rate) per gram. The thermal energy is then perturbed by small changes in density, $\rho=\rho_{u}+\delta \rho$, and pressure, $P=$ $P_{u}+\delta P$, where the subscript $u$ denotes the variables in the unperturbed medium. Then, for short-wave perturbations whose characteristic frequency is considerably greater than the inverse thermal cooling time $\left(t_{\text {cool }}\right)$, we have adiabatic perturbations [65] and the condition that must be verified for the thermal instability is

$$
\frac{1}{\gamma-1} \frac{\rho_{u}}{T_{u}}\left(\frac{\partial L}{\partial \rho}\right)_{T_{u}}+\left(\frac{\partial L}{\partial T}\right)_{\rho_{u}}-\frac{L_{u}}{T_{u}}<0 .
$$

For perturbations of longer wavelength, with characteristic hydrodynamic times comparable to the cooling time, they can 
be considered as isobaric. In this case the criterion for the instability is

$$
\frac{\rho_{u}}{T_{u}}\left(\frac{\partial L}{\partial \rho}\right)_{T_{u}}-\left(\frac{\partial L}{\partial T}\right)_{\rho_{u}}-\frac{L_{u}}{T_{u}}>0 .
$$

Finally, for long-wavelength perturbations, whose characteristic hydrodynamic time is considerably larger than the cooling time, we have isochoric perturbations and the criteria is then given by [65]

$$
\left(\frac{\partial L}{\partial T}\right)_{\rho_{u}}-\frac{L_{u}}{T_{u}}<0 .
$$

From the equations we can observe that the criteria mainly depend on $L$ and its derivatives with respect to temperature and density. We are dealing with radiative shocks in which the radiative cooling becomes a dominant process. For this reason, we can neglect the heating rate in $L$ and consider only the radiative cooling rate per gram. The radiative cooling rate is obtained from the divergence of the radiative flux that is given by Eq. (39). We have already obtained that in the range of plasma conditions under analysis we can approximate this quantity to the radiative power loss and assume that $L \approx \mathrm{RPL} / \rho$. For radiative shocks it is usual to fit the RPL to a power law of the temperature and the density of matter. Most of these fittings are made assuming that RPL $=\rho^{2} \Lambda(T)$, where $\Lambda(T)$ is the cooling function, with $\Lambda(T) \propto T^{\beta}$. This approach is valid when the bremsstrahlung is the dominant radiative process or when the plasma can be considered in corona equilibrium, but neither of these requirements is fulfilled in the plasma conditions of this work. For this reason, we have locally fitted the RPL by the power law $C \rho^{\alpha} T^{\beta}$ using the PARPRA code [70] and imposing a maximum relative error of $0.1 \%$ in the fitting. By substituting this expression for the RPL and $L$ into Eqs. (41)-(43) we obtain the criteria for the thermal instabilities in terms of the cooling power as

$$
\beta< \begin{cases}1 & \text { isochoric } \\ \alpha & \text { isobaric } \\ \frac{\gamma-\alpha}{\gamma-1} & \text { adiabatic. }\end{cases}
$$

We have already used our fitting of the RPL and these criteria to predict the possibility of isobaric radiative cooling instabilities experiments of convergent radiative shocks in argon and neon generated in a cylindrical liner $Z$-pinch configuration, obtaining results that are consistent with the experimental observations [71].

In Fig. 23 we have represented the parameter $\beta$ and the three criteria for the thermal instabilities against the temperature and for the density of matter in the cooling layer. From the figure we detect two regions of temperatures in which the criteria are fulfilled, $12-15$ and $22-28 \mathrm{eV}$, respectively. As said before, in our case the temperatures in postshock are between 12 and $17 \mathrm{eV}$ and thermal instabilities may arise in that region, according to Fig. 23. We have also made the analysis for the lowest and largest matter densities of the range considered in this work. In both cases, two regions of temperatures where the instabilities can occur are also obtained. As the density increases the two regions are shifted toward higher temperatures (13-17 and 23-31 eV, respectively,

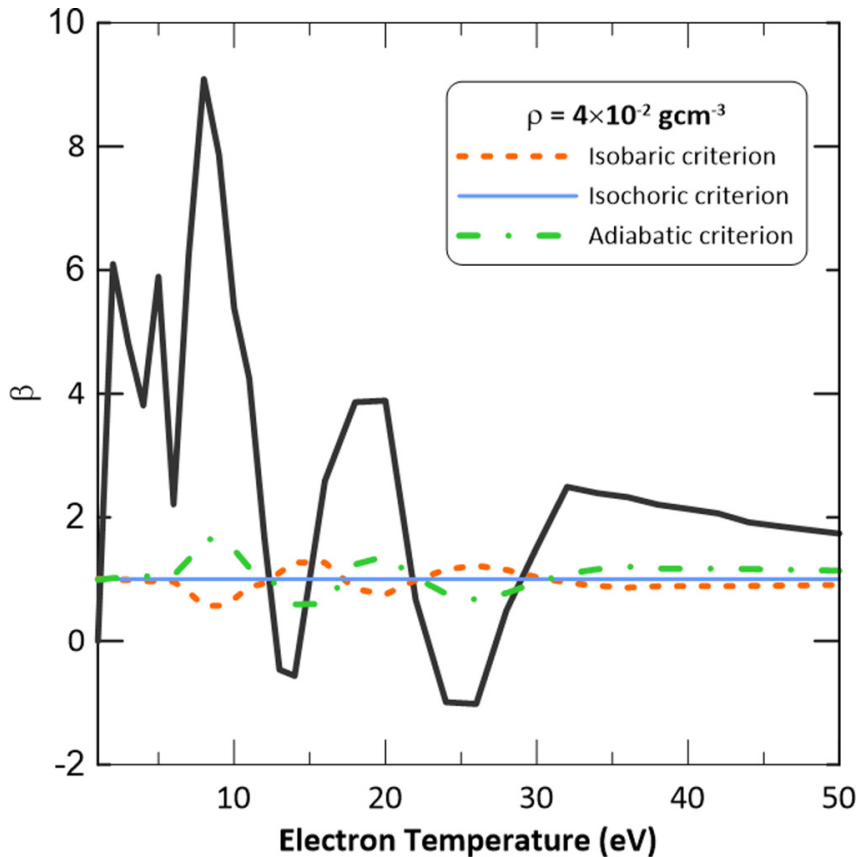

FIG. 23. (Color online) Values of the exponent of the temperature, $\beta$ (black solid line), as a function of the temperature and the three criteria for thermal instabilities.

at $\left.10^{-3} \mathrm{~g} \mathrm{~cm}^{-3}\right)$, while the opposite is happening as the density decreases (8-12 and 17-22 eV, respectively, at $10^{-3} \mathrm{~g} \mathrm{~cm}^{-3}$ ).

Langer et al. [66] proposed theoretically a new kind of thermal instability while modeling the accretion of matter on to stellar surfaces. They discovered that the cooling region can be subject to oscillations due to the onset of this kind of thermal instability. Its physical basis is that while the shock is moving out, it heats the gas to a higher temperature, increasing the cooling time with respect to the steady-state case [72] and, as a consequence, the shock structure can maintain a larger cooling region. On the contrary, when the shock wave is moving in, the situation is reversed, leading to amplified oscillations, i.e., overstability [73]. The analysis of the onset of the oscillatory instability is made in terms of the cooling law of the gas. Langer et al. [66] assumed RPL $\propto \rho^{2} T^{1 / 2}$, i.e., bremsstrahlung cooling. Chevalier and Imamura [72] performed a linear analysis with RPL $\propto \rho^{2} T^{\beta}$. Imamura et al. [74] presented a linear analysis for RPL $\propto$ $\rho^{\alpha} T^{\beta}$, showing that the instability criterion depends more on $\alpha-\beta$ than on $\alpha$ or $\beta$ separately [73]. We have previously employed this model using the RPLs obtained with our collisional-radiative model to analyze the possibility of this kind of instability in blast waves launched in clusters of krypton and xenon, obtaining results that are consistent with the experimental observations [26].

In this work the cooling law is RPL $\propto \rho^{\alpha} T^{\beta}$ and for the study of the oscillatory instability we have followed the analysis performed by Ramachandran and Smith [73]. They consider a similar cooling law and study atomic gas impacting against a rigid wall in $1 \mathrm{D}$, a situation that might be analogous to our experiment. From our fitting we have obtained that $\alpha \approx 1$ for the whole range of temperatures considered, overall as the density of matter increases. When the energy levels 
of the atoms which provide the dominant cooling are in LTE then $\alpha=1$ [73]. We showed in Sec. III that for low temperatures in the low-density regime and for the whole range of electron temperatures for larger densities the plasma could be considered in LTE and this fact explains why we obtain $\alpha \approx 1$ in our power law for the RPL. Taking into account that $\gamma=5 / 3$, the overstability sets in for values of $\beta$ below $0.051,0.149,0.028$, and 0.147 (the fundamental, first, second, and third overtones, respectively) according to Ref. [73]. For the experiment under analysis, the regions of temperatures in which the previous criteria are fulfilled are 13-14 eV and 24-26 eV as Fig. 23 shows. The temperatures obtained in the hydrodynamic simulation for the cooling layer are in these ranges and, therefore, the medium could be unstable.

The hydrodynamic simulation using MULTI (1D) shows neither of the thermal instabilities commented above. On the other hand, in a 2D simulation performed using the ARWEN code [75] instabilities are detected, although it is not clear that they are due to radiative cooling. Unfortunately, there are not enough experimental images that allow us to conclude whether there are thermal instabilities in this experiment. However, as noted above, we have already successfully applied our analysis of thermal instabilities to other experiments on radiative shocks and, therefore, we expect that our predictions for this experiment will be correct as well.

\section{CONCLUSIONS}

In this work we have analyzed the microscopic properties of xenon plasmas in ranges of matter densities $\left(10^{-3}-10^{-1} \mathrm{~g} \mathrm{~cm}^{-3}\right)$ and electron temperatures $(1-50 \mathrm{eV})$ typically found in EUV lithography and some laboratory astrophysical experiments such as those related to the study of radiative shocks. In particular, we have studied the effect of both theoretical approaches and the influence of plasma self-absorption and external radiation fields in the calculation of the plasma-level populations and radiative properties. Thus, making use of a CRSS model, where accuracy for the simulation of microscopic properties of xenon plasmas is previously checked, we have analyzed the validity of the LTE assumption for that range of plasma conditions. We have obtained that for the densities between $10^{-2}$ and $10^{-1} \mathrm{~g} \mathrm{~cm}^{-3}$ and for the whole range of electron temperatures LTE assumption is accurate to calculate the plasma radiative properties. On the other hand, as the density decreases the maximum value of the electron temperature where LTE can be assumed also does. We have also analyzed the influence of the atomic description by means of the comparison among the radiative calculated using DCA, DLA, an DLA+CI atomic approaches. We have found that the DLA description does not introduce significant improvements with respect to the DCA description, whereas the configuration interaction effects are considerable in both monochromatic and mean radiative properties. We have also made in this work a study of the influence of plasma self-absorption and external radiation field in the calculation of the plasma-level populations. In particular, this study was made for the radiative precursor generated in a experiment of a radiative shock launched in xenon carried out at PALS. Plasma self-absorption was modeled assuming the plasma with planar geometry and making use of the escape factor formalism to take into account the opacity effects in the population kinetic calculations. We found that plasma self-absorption effects in the monochromatic opacities and emissivities were observable mainly for the regions of the radiative precursor nearest to the shock front where the temperature in the precursor is higher. On the other hand, the effect on the mean properties, even in those regions, is small. Therefore, in principle, for those calculations that do not require monochromatic radiative properties, it seems that including self-absorption effects would not be necessary. However, in order to analyze experimental spectra, they should be considered. The influence of the radiation field on the simulation of the microscopic properties of the precursor was analyzed, including both the radiation field coming from the shock front and the one due to plasma self-emission in the precursor. We detected that the latter is very relevant since it compensates the absorption of the radiation coming from the shock front in some ranges of photon energies. We made a qualitative analysis of the influence of the radiation in the CRSS simulations approaching the external radiation field by a diluted Planck function. We found that the plasma microscopic properties significantly change when the radiation is included in the simulation. The differences decrease as the distance in the precursor from the shock front increases, and this may be due to the diminution of the incoming radiation and of the electron temperature in the precursor that encourages the LTE regime, i.e., the collisional processes. In any case, in this work we have only made a qualitative analysis of the effect and a more accurate calculation could require a more properly modeling of the radiation field. Finally, we have analyzed the possibility of thermal instabilities in this experiment due to radiative cooling in the cooling layer and of thermal oscillations (overstability) in the shock front. This study was made in terms of a power-law fitting of the radiative power loss. We found that for the density of matter in the postshock region and the temperatures provided by the hydrodynamic simulation for this region, the criteria for isochoric, isobaric, and adiabatic instabilities are fulfilled and then they could occur, depending on the relation between the hydrodynamic characteristic time of the perturbation and the cooling time. The criteria for the fundamental and the three first overtones for the overstability of the shock front are also fulfilled and then this thermal instability could also arise. It is clear from this work that some aspects such as the population kinetic model (e.g., LTE or NLTE, plasma self-absorption, external radiation field) or the atomic description selected might affect the calculation of the plasma-level populations and radiative properties in the range of plasma conditions analyzed. However, the inclusion of such often involve a considerable increase of the computational times and this fact must be appropriately assessed depending on the kind of simulation in which we are interested.

\section{ACKNOWLEDGMENTS}

This work was supported by the EURATOM within the ToIFE activities. 
[1] F. Suzuki-Vidal, M. Bochhi, S. Lebedev, G. Swandling, G. Burdiak, S. Bland, P. de Grouchy, G. Hall, E. HarveyThomsom, E. Khoory, S. Pantakar, L. Pickworth, J. Skidmore, R. Smith, J. Chittenden, M. Krishnan, R. Maden, K. Wilson-Elliot, A. Ciardi, and A. Frank, Phys. Plasmas 19, 022708 (2012).

[2] D. Ryutov, R. Drake, J. Kane, E. Liang, B. Remington, and W. Wood-Vasey, Astrophys. J. 518, 821 (1999).

[3] D. Ryutov, B. Remington, H. Robey, and R. Drake, Phys. Plasmas 8, 1804 (2001).

[4] B. A. Remington, R. P. Drake, and D. D. Ryutov, Rev. Mod. Phys. 78, 755 (2006).

[5] J. Castor, Astrophys. Space Sci. 307, 207 (2007).

[6] E. Falize, D. Bouquet, and C. Michaut, Astrophys. Space Sci. 322, 107 (2009).

[7] S. Bouquet, E. Falize, C. Michaut, C. Gregory, B. Loupias, T. Vinci, and M. Koenig, High Energy Density Phys. 6, 368 (2010).

[8] A. Moore, J. Lazarus, M. Hohenberger, J. Robinson, E. Gumbrell, M. Dunne, and R. Smith, Astrophys. Space Sci. 307, 139 (2007).

[9] R. Drake, Phys. Plasmas 14, 043301 (2007).

[10] R. Drake, High Energy Density Physics: Fundamentals, Inertial Fusion and Experimental Astrophysics (Springer, Berlin, 2005).

[11] A. Reighard, R. Drake, K. Dannenberg, D. Kremer, M. Grosskopf, E. Harding, D. Leibrandt, S. Glendinning, T. Perry, B. Remington, J. Greenough, J. Knauer, T. Boehly, S. Bouquet, L. Boireau, M. Koenig, and T. Vinci, Phys. Plasmas 13, 082901 (2006).

[12] F. Doss, R. Drake, and C. Kuranz, High Energy Density Phys. 6, 157 (2010).

[13] R. Drake, F. Doss, R. McClarren, M. Adams, N. Amato, D. Bingham, C. Chou, C. DiStefano, K. Fidkowski, B. Fryxell, T. Gombosi, M. Grosskopf, J. Holloway, B. Van der Holst, C. Huntington, S. Karni, C. Krauland, C. Kuranz, E. Larsen, B. van Leer, B. Mallick, D. Marion, W. Martin, J. Morel, E. Myra, V. Nair, K. Powell, L. Rauchwerger, P. Roea, E. Rutter, I. Sokolov, Q. Stout, B. Torralva, G. Toth, K. Thornton, and A. Visco, High Energy Density Phys. 7, 130 (2011).

[14] B. Fryxell, E. Rutter, and E. Myra, High Energy Density Phys. 8, 141 (2012).

[15] S. Bouquet, C. Stehlé, M. Koenig, J. P. Chieze, A. BenuzziMounaix, D. Batani, S. Leygnac, X. Fleury, H. Merdji, C. Michaut, F. Thais, N. Grandjouan, T. Hall, E. Henry, V. Malka, and J. P. J. Lafon, Phys. Rev. Lett. 92, 225001 (2004).

[16] M. Koenig, T. Vinci, A. Benuzzi-Mounaix, N. Ozaki, A. Ravasio, M. le Glohaec, L. Boireau, C. Michaut, S. Bouquet, S. Atzeni, A. Schiavi, O. Peyrusse, and D. Batani, Phys. Plasmas 13, 056504 (2006).

[17] A. Benuzzi-Mounaix, M. Koenig, A. Ravasio, T. Vinci, N. Ozaki, M. Rabec le Gloahec, B. Loupias, G. Huser, E. Henry, S. Bouquet, C. Michaut, D. Hicks, A. MacKinnon, P. Patel, H. Park, S. Le Pape, T. Boehly, M. Borghesi, C. Cecchetti, M. Notley, R. Clark, S. Bandyopadhyay, S. Atzeni, A. Schiavi, Y. Aglitskiy, A. Faenov, T. Pikuz, D. Batani, R. Dezulian, and K. Tanaka, Plasma Phys. Control. Fusion 48, B347 (2006).

[18] E. Falize, A. Ravasio, B. Loupias, A. Diziere, C. Gregory, C. Michaut, C. Busschaert, C. C., and M. Koenig, High Energy Density Phys. 8, 1 (2012).

[19] J. Grun, J. Stamper, C. Manka, J. Resnick, R. Burris, J. Crawford, and B. H. Ripin, Phys. Rev. Lett. 66, 2738 (1991).
[20] A. D. Edens, T. Ditmire, J. F. Hansen, M. J. Edwards, R. G. Adams, P. K. Rambo, L. Ruggles, I. C. Smith, and J. L. Porter, Phys. Rev. Lett. 95, 244503 (2005).

[21] T. Ditmire, K. Shigemori, B. Remington, K. Estabrook, and R. Smith, Astrophys. J. Suppl. Ser. 127, 299 (2000).

[22] K. Keilty, E. Liang, T. Ditmire, B. Remington, K. Shigemori, and A. Rubenchick, Astrophys. J. 538, 645 (2000).

[23] D. Symes, J. Osterhoff, R. Faustlin, M. Maurer, A. Bernstein, A. Moore, E. Gumbrell, A. Edens, R. Smith, and T. Ditmire, High Energy Density Phys. 3, 353 (2007).

[24] J. Osterhoff, D. Symes, A. Edens, A. Moore, E. Hellewell, and T. Ditmire, New J. Phys. 11, 023022 (2009).

[25] M. Busquet, M. Klapisch, and F. Thais, High Energy Density Phys. 7, 217 (2011).

[26] R. Rodríguez, J. M. Gil, G. Espinosa, R. Florido, J. Rubiano, M. A. Mendoza, P. Martel, E. Mínguez, D. Symes, M. Hohenberger, and R. Smith, Plasma Phys. Control. Fusion 54, 045012 (2012).

[27] J. Zeng, C. Gao, and J. Yuan, Eur. Phys. J. D 60, 309 (2010).

[28] F. Gilleron, M. Poirier, T. Blenski, M. Schmidt, and T. Ceccotti, J. Appl. Phys. 94, 2086 (2003).

[29] N. Bowering, M. Martins, W. Partlo, and I. Fomenkov, J. Appl. Phys. 95, 16 (2004).

[30] T. Krucken, K. Bergmann, L. Juschin, and R. Lebert, J. Phys. D: Appl. Phys. 37, 3213 (2004).

[31] A. Sasaki, K. Nishihara, F. Koike, T. Kagawa, T. Nishikawa, K. Fujima, T. Kawamura, and H. Furukawa, IEEE J. Sel. Top. Quant. 10, 1307 (2004).

[32] E. R. Kieft, K. Garloff, J. J. A. M. van der Mullen and V Banine, Phys. Rev. E 71, 036402 (2005).

[33] C. Stehlé, M. Kozlova, J. Larour, J. Nejdl, N. Champion, P. Barroso, F. Suzuki-Vidal, O. Acef, J. Delattre, P. A. Dostal, M. Krus, and J. Chieze, Opt. Commun. 285, 64 (2012).

[34] M. F. Gu, Can. J. Phys. 86, 675 (2008).

[35] J. Bauche, C. Bauche-Arnoult, and M. Klapisch, Adv. At. Mol. Phys. 23, 131 (1988).

[36] J. C. Stewart and K. D. Pyatt, Astrophys. J. 144, 1203 (1966).

[37] R. Florido, R. Rodríguez, J. M. Gil, J. G. Rubiano, P. Martel, E. Mínguez, and R. C. Mancini, Phys. Rev. E 80, 056402 (2009).

[38] W. Lotz, Z. Physik 216, 241 (1968).

[39] H. Van Regemorter, Astrophys. J. 136, 906 (1962).

[40] H. A. Kramers, Philos. Mag. 46, 836 (1923).

[41] H. R. Griem, Principles of Plasma Spectroscopy (Cambridge University Press, Cambridge, UK, 1997).

[42] R. C. Mancini, R. F. Joyce, and C. F. Hooper Jr., J. Phys. B: At. Mol. Phys. 20, 2975 (1987).

[43] R. Rodríguez, R. Florido, J. M. Gil, J. Rubiano, P. Martel, and E. Mínguez, Laser Part. Beams 26, 433 (2008).

[44] R. Rodríguez, R. Florido, J. M. Gil, J. G. Rubiano, D. Suárez, P. Martel, E. Mínguez, and R. C. Mancini, Commun. Comput. Phys. 8, 185 (2010).

[45] D. Mihalas, Stellar Atmospheres (W. H. Freeman and Company, San Francisco, 1978).

[46] M. S. Dimitrijevic and N. Konjevic, Astron. Astrophys. 172, 345 (1987).

[47] S. J. Rose, J. Phys. B: At. Mol. Opt. Phys. 25, 1667 (1992).

[48] R. J. Rutten, Radiative Transfer in Stellar Atmospheres (Sterrekundig Instituut Utrecht, 1995). 
[49] F. Serduke, E. Mínguez, S. Davidson, and C. Iglesias, J. Quant. Spectrosc. Radiat. Transfer 65, 527 (2000).

[50] H. K. Chung, K. B. Fournier, and R. W. Lee, High Energy Density Phys. 2, 7 (2006).

[51] W. Karzas and R. Latter, Astrophys. J. 6, 167 (1961).

[52] E. Mínguez, R. Florido, R. Rodríguez, J. M. Gil, J. Rubiano, M. A. Mendoza, D. Suárez, and P. Martel, High Energy Density Phys. 6, 57 (2010).

[53] J. G. Rubiano, R. Florido, C. Bowen, R. W. Lee, and Y. Ralchenko, High Energy Density Phys. 3, 225 (2007).

[54] A. Bar-Shalom, J. Oreg, W. H. Goldstein, D. Shvarts, and A. Zigler, Phys. Rev. A 40, 3183 (1989).

[55] F. Perrot, T. Blenski, and A. Grimaldi, J. Quant. Spectrosc. Radiat. Transfer 58, 845 (1997).

[56] T. Blenski, A. Grimaldi, and F. Perrot, J. Quant. Spectrosc. Radiat. Transfer 65, 91 (2000).

[57] A. Bar-Shalom, M. Klapisch, and J. Oreg, J. Quant. Spectrosc. Radiat. Transfer 71, 169 (2001).

[58] H. Griem, Handbook of Plasma Physics, edited by M. Rosenbluth and R. Sagdeev, Vol. 1 (North-Holland, Amsterdam, 1983), p. 73.

[59] T.J. Baig and H.J. Kunze, Phys. Rev. E 48, 2103 (1993).

[60] R. Rodríguez, J. M. Gil, R. Florido, J. Rubiano, M. A. Mendoza, P. Martel, and E. Mínguez, Contrib. Plasma Phys. 51, 863 (2011).

[61] C. Bowen, R. W. Lee, and Y. Ralchenko, J. Quant. Spectrosc. Radiat. Transfer 99, 102 (2006).
[62] R. Ramis, R. Schmalz, and J. Meyer-Ter-Vehn, Comp. Phys. Comm. 49, 475 (1988).

[63] G. Field, Astrophys. J. 142, 531 (1965).

[64] J. J. Hunter, Astrophys. J. 161, 451 (1970).

[65] Yu. A. Shchenikov, Sov. Astron. 22, 182 (1978).

[66] S. Langer, G. Chanmugam, and G. Shaviv, Astrophys. J. 245, L23 (1981).

[67] D. Lynden-Bell and C. Tout, Astrophys. J. 558, 1 (2001).

[68] F. Heitsch, L. Hartmann, and A. Burkert, Astrophys. J. 683, 786 (2008).

[69] E. Vasiliev, Mon. Not. Roy. Astron. Soc. 419, 3641 (2012).

[70] R. Rodríguez, G. Espinosa, J. M. Gil, J. G. Rubiano, M. A. Mendoza, P. Martel, and E. Mínguez, Commun. Comput. Phys. 16, 612 (2014).

[71] G. Burdiak, S. Lebedev, F. Suzuki-Vidal, G. Swadling, S. Bland, N. Niasse, L. Suttle, M. Bennet, J. Hare, M. Weinwurm, R. Rodríguez, J. Gil, and G. Espinosa, J. Plasma Phys. 81, 365810301 (2015).

[72] R. Chevalier and J. Imamura, Astrophys. J. 261, 543 (1982).

[73] B. Ramachandran and M. Smith, Mon. Not. R. Astron. Soc. 357, 707 (2005).

[74] J. Imamura, A. Aboasha, M. Wolff, and K. Wood, Astrophys. J. 458, 327 (1996).

[75] M. Cotelo, P. Velarde, A. de la Varga, D. Portillo, C. Stehlé, U. Chaulagain, M. Kozlova, J. Larour, and F. Suzuki-Vidal (to be published). 